\title{
Integrating physiologically based kinetic (PBK) and Monte Carlo modelling to predict inter-individual and inter-ethnic variation in bioactivation and liver toxicity of lasiocarpine
}

\author{
Jia Ning ${ }^{1} \cdot$ Ivonne M. C. M. Rietjens ${ }^{1} \cdot$ Marije Strikwold $^{2}$
}

Received: 12 June 2019 / Accepted: 2 September 2019 / Published online: 11 September 2019

(c) The Author(s) 2019

\begin{abstract}
The aim of the present study was to predict the effect of inter-individual and inter-ethnic human kinetic variation on the sensitivity towards acute liver toxicity of lasiocarpine in the Chinese and the Caucasian population, and to derive chemical specific adjustment factors (CSAFs) by integrating variation in the in vitro kinetic constants $V_{\max }$ and $K_{\mathrm{m}}$, physiologically based kinetic (PBK) modelling and Monte Carlo simulation. CSAFs were derived covering the 90th and 99th percentile of the population distribution of pyrrole glutathione adduct (7-GS-DHP) formation, reflecting bioactivation. The results revealed that in the Chinese population, as compared to the Caucasian population, the predicted 7-GS-DHP formation at the geometric mean, the 90th and the 99th percentile were 2.1-, 3.3- and 4.3-fold lower respectively. The CSAFs obtained using the 99th percentile values were 8.3,17.0 and 19.5 in the Chinese, the Caucasian population and the two populations combined, respectively, while the CSAFs were generally 3.0-fold lower at the 90th percentile. These results indicate that when considering the formation of 7-GS-DHP the Caucasian population may be more sensitive towards acute liver toxicity of lasiocarpine, and further point out that the default safety factor of 3.16 for inter-individual human kinetic differences may not be sufficiently protective. Altogether, the results obtained demonstrate that integrating PBK modelling with Monte Carlo simulations using human in vitro data is a powerful strategy to quantify inter-individual variations in kinetics, and can be used to refine the human risk assessment of pyrrolizidine alkaloids.
\end{abstract}

Keywords Physiologically based kinetic (PBK) model · Monte Carlo simulations · Chemical specific adjustment factors · Lasiocarpine $\cdot$ Pyrrole glutathione adduct $\cdot$ Inter-ethnic

$\begin{array}{ll}\text { Abbreviations } \\ \mathrm{BMD}_{5 / 10} & \begin{array}{l}\text { The benchmark dose resulting in 5\%/10\% } \\ \text { extra effect }\end{array} \\ \mathrm{BMDL}_{5 / 10} & \begin{array}{l}\text { The lower confidence limit of the bench- } \\ \text { mark dose resulting in 5\%/10\% extra effect }\end{array} \\ \mathrm{BMDU}_{5 / 10} & \begin{array}{l}\text { The upper confidence limit of the bench- } \\ \text { mark dose resulting in 5\%/10\% extra effect } \\ \text { Maximum concentration in liver blood }\end{array} \\ C_{\text {max }} & \end{array}$

Electronic supplementary material The online version of this article (https://doi.org/10.1007/s00204-019-02563-x) contains supplementary material, which is available to authorized users.

Jia Ning

jia.ning@wur.nl

1 Division of Toxicology, Wageningen University, Stippeneng 4, 6708 WE Wageningen, The Netherlands

2 Van Hall Larenstein University of Applied Sciences, PO Box 1528, 8901 BV Leeuwarden, The Netherlands
CSAF
EFSA
Chemical specific adjustment factor
IARC
European Food Safety Authority
IPCS The International Programme on Chemical
The International Agency for Research on Cancer
NOAEL No observed adverse effect level
PAs Pyrrolizidine alkaloids
PBK model Physiologically based kinetic model
PoD(s) Point(s) of departure
UPLC Ultra performance liquid chromatography

\section{Introduction}

Lasiocarpine is one of the 1,2-unsaturated pyrrolizidine alkaloids (PAs) which are secondary metabolites of certain flowering plants. Lasiocarpine is widely distributed in the Heliotropium and Symphytum genus (Smith and Culvenor 
1981). Human exposure to lasiocarpine may result from consumption of contaminated staple foods, plant food supplements, herbal teas and herbal medicines (EFSA 2017, 2011). Exposure to lasiocarpine is known to cause acute liver toxicity in humans. The latest documented outbreak has been reported in Afghanistan in 2008, where 38 people suffered from massive ascites and four people died due to consumption of wheat flour contaminated with seeds of Heliotropium, containing lasiocarpine (EFSA 2011; Kakar et al. 2010). Next to acute liver toxicity, lasiocarpine is also classified as a Group 2B carcinogen due to its carcinogenicity induced by a genotoxic mode of action (IARC 2017; NTP 1978).

It is generally accepted that lasiocarpine exerts its hepatotoxicity and carcinogenicity upon metabolic activation (Fu et al. 2004; IPCS 1989; Prakash et al. 1999; Stegelmeier et al. 1999). Three major metabolic pathways of lasiocarpine can be distinguished, namely detoxification via (1) hydrolysis of lasiocarpine into a necine base or (2) $\mathrm{N}$-oxidation to lasiocarpine $\mathrm{N}$-oxide, and bioactivation by (3) hydroxylation at the $\mathrm{C}-3$ or $\mathrm{C}-8$ position of the necine base, followed by dehydration into dehydrolasiocarpine (Fig. 1). Dehydrolasiocarpine is highly reactive and is able to attack nucleophilic molecules such as DNA and proteins resulting in hepatotoxicity and carcinogenicity (Fu et al. 2004). A recent in vitro study using human liver microsomes provided convincing evidence that the metabolism of lasiocarpine is mainly catalyzed by the CYP 3A enzyme family, particularly CYP 3A4 (Fashe et al. 2015). Other studies also reported that PA metabolism is mainly catalyzed by the CYP $3 \mathrm{~A} 4$ enzyme in human (Fu et al. 2004; Li et al. 2011; Miranda et al. 1991).

It is well known that the abundance and activity of CYP $3 \mathrm{~A} 4$ in the liver varies significantly in individuals and in different ethnic groups (Fu et al. 2004). Some studies reported that the variation in CYP $3 \mathrm{~A} 4$ expression including mRNA and protein levels in the liver can be up to 100-fold and even 400-fold when taking illness, inhibition and induction-related interactions of drugs into account (WestlindJohnsson et al. 2003; Wilkinson 2005). This large variation in CYP 3A activity may result in marked variations in the metabolic profile of PAs and thus different susceptibilities towards pyrrolizidine alkaloid intoxication (Fu et al. 2004). Therefore, human inter-individual and inter-ethnic (Chinese and Caucasian) variation upon PA metabolism may be higher than the default uncertainty factor of 3.16 for interindividual human kinetic differences (IPCS 2005; Bhat et al. 2017) that is used to derive health-based guidance values for the risk assessment of compounds with non-genotoxic critical effects, indicating the need for a chemical specific adjustment factor (CSAF) (Bhat et al. 2017). For risk assessment of compounds that are both genotoxic and carcinogenic, the default uncertainty factor of 3.16 for inter-individual human

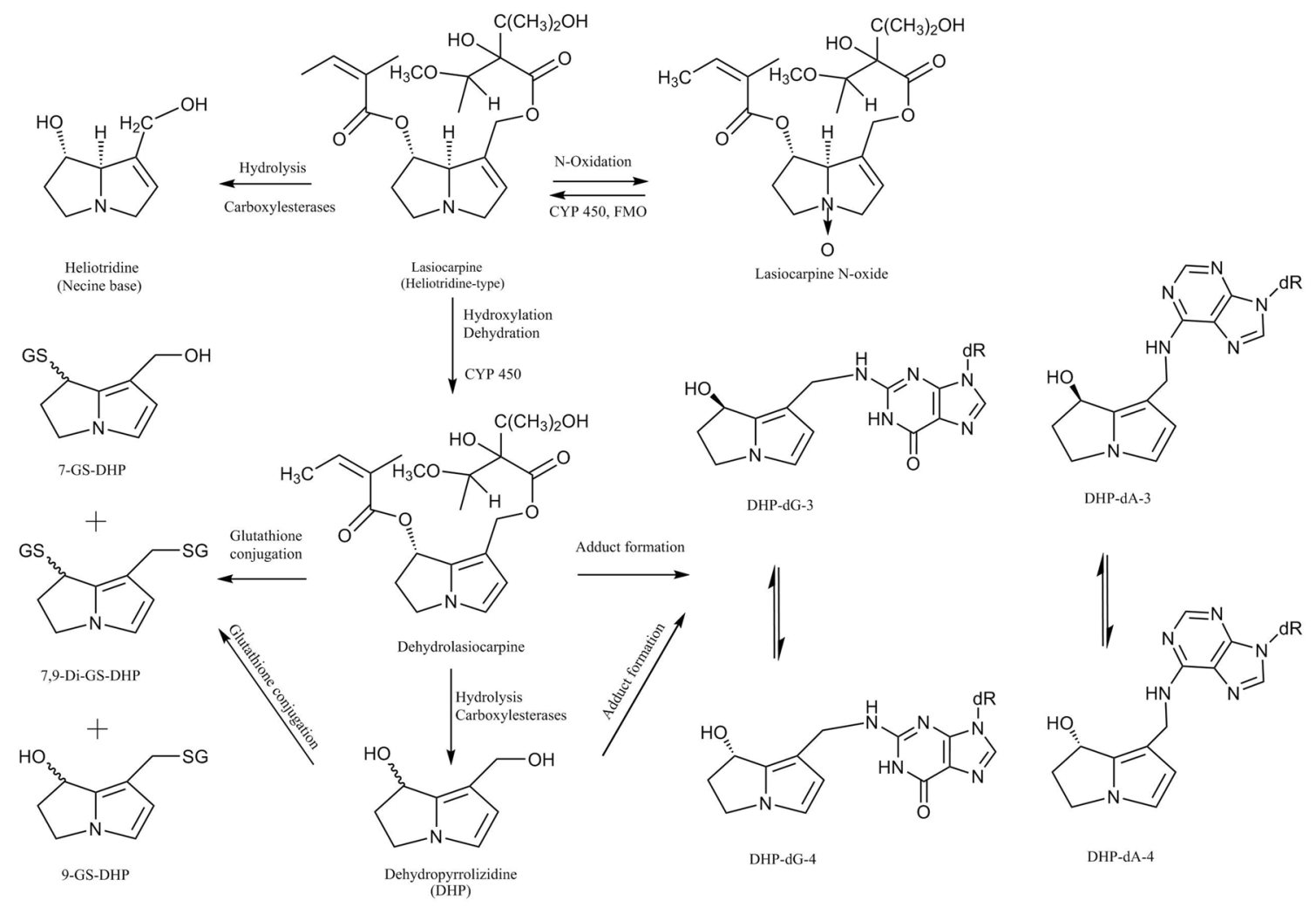

Fig. 1 Principal metabolism pathways of lasiocarpine in humans. FMO flavin-containing monooxygenase, $C Y P 450$ cytochromes $\mathrm{P} 450$ 
kinetic differences is part of the cut-off value of 10,000 for evaluating the margin of exposure (MOE) (EFSA 2005, 2012, 2015).

Previously, we predicted the inter-ethnic differences in acute liver toxicity between the average Chinese and Caucasian subject to amount to 2-fold for lasiocarpine with the average Caucasian being more sensitive than the average Chinese. This was done by applying physiologically based kinetic (PBK) modelling-based reverse dosimetry translating in vitro hepatic cytotoxicity data obtained in primary hepatocytes in vitro to in vivo acute liver toxicity of lasiocarpine and riddelliine for the average Chinese population and the average Caucasian population. Mixed gender pooled Chinese and Caucasian liver fractions were used to predict the kinetic constants for metabolism of these compounds in the liver which were subsequently included in the PBK models for the average Chinese and Caucasians (Ning et al. 2019). The PBK model of lasiocarpine for human was evaluated mainly based on the evaluation of the same model of riddelliine for human in our previous study where the predicted $\mathrm{BMD}_{5}$ values of liver toxicity for riddelliine in Chinese and Caucasian subjects amounting to $2.5 \mathrm{mg} / \mathrm{kg}$ bw/day and $0.5 \mathrm{mg} / \mathrm{kg}$ bw/ day, respectively (Ning et al. 2019) were comparable to the reported in vivo liver toxicity data for human amounting to $0.7-3 \mathrm{mg} / \mathrm{kg}$ bw/day (Culvenor 1983). In addition, the PBK models developed for lasiocarpine for the Chinese and Caucasian population were developed based on PBK models previously defined and validated in rats, shown to adequately predict the liver toxicity of lasiocarpine in rats based on toxicity data in rat hepatocytes (Chen et al. 2018). However, our previous study did not characterize the inter-individual variations within the Chinese and the Caucasian population. Given the large variation among individuals in terms of CYP 3A4 variability, the aim of the present study was to study the effect of inter-individual and inter-ethnic (Chinese and Caucasian) kinetic variation on sensitivity towards bioactivation and acute liver toxicity of lasiocarpine using liver fractions from 25 male Chinese individuals and 25 male Caucasian individuals, and to derive CSAFs by applying PBK modelling together with Monte Carlo simulation.

\section{Materials and methods}

\section{Compounds and biological materials}

Lasiocarpine (purity $>97 \%$ ) was purchased from Phytolab (Vestenbergsgreuth, Germany). Dehydromonocrotaline (purity $\geq 90 \%$ ) was purchased from Santa Cruz Biotechnology (Heidelberg, Germany). Potassium dihydrogen phosphate, trifluoroacetic acid (TFA) and formic acid were purchased from VWR International (Darmstadt, Germany). Phosphate buffered saline (PBS) was purchased from Gibco
(Paisley, Scotland, UK). Phosphoric acid was obtained from Merck (Darmstadt, Germany). Reduced nicotinamide adenine dinucleotide phosphate (NADPH) was obtained from Roche Diagnostics (Mannheim, Germany). Acetonitrile (UPLC/MS grade) was obtained from Biosolve BV (Valkenswaard, The Netherlands). Glutathione (GSH), N,Ndimethylformamide (DMF) and dimethylsulfoxide (DMSO) were purchased from Sigma-Aldrich (Steinheim, Germany). Twenty-five individual male human liver microsomes of Caucasian origin were purchased from XenoTech (Lenexa, USA). Twenty-five individual male human liver microsomes of Chinese origin were purchased from PrimeTox (Wuhan, China). The characteristics of the human liver microsome donors are presented in Table $\mathrm{S} 1$ in the supplementary materials 1 .

\section{General outline for PBK modelling with Monte Carlo simulation}

The outline of the present study consisted of the following steps: (1) the metabolism of lasiocarpine in the liver was studied using subcellular liver fractions of 25 male Chinese individuals and 25 male Caucasian individuals. From this the coefficient of variation $(\mathrm{CV})$ values of lasiocarpine depletion and 7-GS-DHP formation were derived, (2) the inter-individual variation in the Chinese and Caucasian population in the kinetic constants $V_{\max }$ and $K_{\mathrm{m}}$ of lasiocarpine depletion and 7-GS-DHP formation were included in the PBK model in connection with Monte Carlo simulations to predict inter-ethnic and inter-individual variation of 7-GSDHP formation by the liver, (3) CSAFs were derived from the results of the Monte Carlo simulations for the Caucasian population, the Chinese population and the two populations combined covering the 90th and 99th percentile of the population distribution of 7-GS-DHP formation in the liver compartment (IPCS 2005), (4) finally, the PBK modelling-based reverse dosimetry approach was used to convert in vitro liver toxicity data obtained in human hepatocytes to in vivo dose-response curves for acute liver toxicity for the average and the sensitive (90th and 99th percentile) individuals in the Chinese and Caucasian populations and the two populations combined.

\section{In vitro microsomal incubations of lasiocarpine depletion and 7-GS-DHP formation}

The in vitro incubations for lasiocarpine depletion and formation of 7-GS-DHP by 25 individual male Chinese and 25 individual male Caucasian liver microsomes were performed according to the method described by Ning et al. (2019). Briefly, individual male liver microsomes were incubated with lasiocarpine in the presence of NADPH either in the absence or presence of GSH. The incubation mixtures 
consisted of $0.1 \mathrm{M}$ potassium phosphate ( $\mathrm{pH} 7.4$ adjusted by phosphoric acid) containing (final concentrations) $0.04 \mathrm{mg} /$ $\mathrm{ml}$ microsomal protein and $2 \mathrm{mM}$ NADPH. Under these incubation conditions conversion of lasiocarpine was linear in time and with the amount of microsomal protein (data not shown). In the final incubations, the substrate concentrations ranged from 3 to $200 \mu \mathrm{M}$ lasiocarpine (added from 100 times concentrated stock solutions in DMSO). The reactions were started after a $5 \mathrm{~min}$ pre-incubation at $37^{\circ} \mathrm{C}$ in a shaking water bath by adding NADPH. Incubations were performed for $30 \mathrm{~min}$ in a shaking water bath at $37{ }^{\circ} \mathrm{C}$. The reactions were terminated by adding $20 \%$ (v/v) ice-cold acetonitrile and the samples were subsequently put on ice. Blank incubations were performed in the absence of the cofactor NADPH for each substrate concentration. Each incubation was performed in duplicate. All samples were centrifuged for $5 \mathrm{~min}$ at $16,000 \mathrm{~g}$ and the supernatants were directly analyzed by UPLC-PAD (Ultra Performance Liquid ChromatographyPhotodiode Array Detector).

The incubation conditions for 7-GS-DHP formation were similar to the conditions to measure lasiocarpine depletion outlined above, with the exception that $4 \mathrm{mM} \mathrm{GSH}$ (final concentration) was added to the incubation mixtures before the $5 \mathrm{~min}$ pre-incubation. The level of GSH in the incubations was chosen to obtain the maximal scavenging of dehydrolasiocarpine (DHPA) and/or of dehydropyrrolizidine (DHP) forming 7-GS-DHP as the major detectable glutathione conjugate. Each incubation was performed in duplicate. All samples were centrifuged for $5 \mathrm{~min}$ at $16,000 \mathrm{~g}$ and the supernatants were directly analyzed by LC-MS/MS.

\section{UPLC analysis of lasiocarpine depletion and LC-MS/ MS analysis of 7-GS-DHP formation}

From each incubation, $3.5 \mu \mathrm{l}$ of supernatant from incubations for determination of depletion of lasiocarpine were injected into the UPLC (Acquity, Waters) using a BEH C18 $(1.7 \mu \mathrm{m} 2.1 \times 50 \mathrm{~mm})$ column with a guard column (Acquity, Waters) and a PAD (Acquity, Waters). The flow rate was $0.6 \mathrm{ml} / \mathrm{min}$ and the mobile phase consisted of ultrapure water with $0.1 \%(\mathrm{v} / \mathrm{v})$ TFA and acetonitrile. For the analysis of lasiocarpine, a gradient was applied from 0 to $50 \%$ acetonitrile in ultrapure water in $2 \mathrm{~min}$, after which the percentage of acetonitrile was increased to $100 \%$ in 3 min and kept at this level for $2 \mathrm{~min}$, after which the starting conditions were reset. Under these conditions, lasiocarpine had a retention time of $1.9 \mathrm{~min}$. Identification of lasiocarpine was achieved by comparison of the UV spectrum and retention time of this compound to those of the reference compound. Quantification of lasiocarpine was based on its peak area measured at a wavelength of $220 \mathrm{~nm}$, using a linear calibration curve $\left(R^{2}=0.999\right)$ of the corresponding standard compound
[1.25-200 $\mu \mathrm{M}$ lasiocarpine added from 100 times concentrated stock solutions in DMSO and further dissolved in $0.1 \mathrm{M}$ potassium phosphate buffer ( $\mathrm{pH}$ 7.4)].

The glutathione conjugate of lasiocarpine, 7-GS-DHP was identified and quantified by LC-MS/MS as described previously (Ning et al. 2019). Briefly, LC-MS/MS analysis was performed on a Shimadzu Nexera XR LC-20AD SR UHPLC system coupled with a Shimadzu LCMS-8040 mass spectrometer. Aliquots of the incubation mixture of $1 \mu \mathrm{l}$ were loaded on a reverse phase $\mathrm{C} 18$ column (Phenomenex $1.7 \mu \mathrm{m} 2.1 \times 50 \mathrm{~mm}$ ). The flow rate was $0.3 \mathrm{ml} / \mathrm{min}$ and the mobile phase was made with ultrapure water with $0.1 \%(\mathrm{v} / \mathrm{v})$ formic acid and acetonitrile containing $0.1 \%(\mathrm{v} / \mathrm{v})$ formic acid. A linear gradient was applied from 0 to $5 \%$ acetonitrile over $8 \mathrm{~min}$ and further increased to $100 \%$ in $6 \mathrm{~min}$. This percentage was kept for $0.5 \mathrm{~min}$, and then reduced to the starting conditions in $0.1 \mathrm{~min}$. Under these conditions, 7-GSDHP eluted at $8.97 \mathrm{~min}$. For MS-MS analysis, a Shimadzu LCMS-8040 triple quadrupole with electrospray ionization (ESI) interface was used. The instrument was operated in positive ionization mode in the multiple reaction monitoring (MRM) mode with a spray voltage of $4.5 \mathrm{kV}$. The 7-GSH-DHP was monitored at the $[\mathrm{M}+\mathrm{H}]^{+}$of precursor to products of $443.2 \rightarrow 425.15(\mathrm{CE}=-7 \mathrm{eV}), 443.2 \rightarrow 118.1$ $(\mathrm{CE}=-24 \mathrm{eV})$ and $443.2 \rightarrow 247.2(\mathrm{CE}=-15 \mathrm{eV}) \mathrm{m} / \mathrm{z}$. The peak area of the total ion chromatogram (TIC) for the 7-GS-DHP formation was used for quantification (Ning et al. 2019).

Quantification of 7-GS-DHP was achieved by comparing the peak area of the 7-GS-DHP formed in the incubation samples to the calibration curve of 7-GS-DHP $\left(r^{2}=0.991\right)$ ranging from 0.078 to $10 \mu \mathrm{M}$ at LC-MS/MS. 7-GS-DHP was synthesized based on the method described by Ma et al. (2015). Briefly, GSH (6.5 mg, $21 \mathrm{mM})$ in $70 \mu \mathrm{l}$ PBS was added to dehydromonocrotaline $(10 \mathrm{mg}, 31 \mathrm{mM})$ in $1 \mathrm{ml}$ DMF with molar ratio of dehydromonocrotaline:GSH of 1:0.67. The reaction mixture was stirred at ambient temperature for $2 \mathrm{~h}$ and quenched with $10 \mathrm{ml}$ PBS. The reaction product was purified by preparative HPLC (Waters, Milford, MA) equipped with an Alltima C18 $5 \mu \mathrm{m}$ column $(150 \times 4.6 \mathrm{~mm})$ and a guard column $(7.5 \times 4.6 \mathrm{~mm})$ (Alltech, Bergen op Zoom, The Netherlands). Detection was performed between 200 and $350 \mathrm{~nm}$ using a Waters 2996 PAD (Waters, Milford, MA). The flow rate was $1 \mathrm{ml} / \mathrm{min}$. The mobile phase used for purification consisted of (A) $0.01 \%$ formic acid in ultrapure water and (B) acetonitrile. A gradient was applied from 5\% B with a linear increase to $15 \%$ in $40 \mathrm{~min}$, after which the gradient returned to the initial condition in $5 \mathrm{~min}$ and remained $5 \mathrm{~min}$ at this condition before the next injection. Figure $\mathrm{S} 2$ in the supplementary materials 2 shows the HPLC-PAD chromatogram of reaction products before purification. The peaks of 7-GS-DHP were collected and subsequently freeze dried. The purity of 7-GS-DHP was 
checked by HPLC-PAD and was determined to be $82 \%$ by comparing the peak area of 7-GS-DHP and the peak area of the impurities after purification. The standard was dissolved in water and aliquots were stored in $-80^{\circ} \mathrm{C}$.

\section{Determination of kinetic constants of lasiocarpine depletion and 7-GS-DHP formation}

The depletion of lasiocarpine was quantified as the amount detected in the blank incubations performed in the absence of the cofactor NADPH minus the amount detected in the full corresponding incubations with NADPH. The amount of 7-GS-DHP formed in the full incubations in the presence of NADPH and GSH was corrected for the amounts detected in the blank incubations without NADPH for each substrate concentration. The data for the rate of conversion of lasiocarpine and 7-GS-DHP formation with increasing substrate concentration $[S]$ were fitted to the standard Michaelis-Menten equation:

$v=V_{\max } /\left(1+K_{\mathrm{m}} /[S]\right)$.

The apparent maximum velocity $\left(V_{\max }\right)$ and the apparent Michaelis-Menten constant $\left(K_{\mathrm{m}}\right)$ were determined by fitting the data to the Michaelis-Menten model using GraphPad (GraphPad Prism software version 5.04, San Diego California USA). The catalytic efficiency in vitro expressed in $\mu \mathrm{l} /$ $\mathrm{min} / \mathrm{mg}$ liver microsomes was calculated as the $V_{\max }$ in vitro divided by the $K_{\mathrm{m}}$. In vitro derived $V_{\max }$ values expressed in $\mathrm{nmol} / \mathrm{min} / \mathrm{mg}$ microsomal protein were scaled to in vivo $V_{\max }$ values expressed in $\mu \mathrm{mol} / \mathrm{h} / \mathrm{g}$ liver using a microsomal protein yield of $35 \mathrm{mg} / \mathrm{g}$ human liver (Medinsky et al. 1994). The $K_{\mathrm{m}}$ values in vitro were assumed to be equal to in vivo $K_{\mathrm{m}}$ values.

\section{PBK modelling}

The PBK model previously developed for lasiocarpine in Chinese and Caucasians was used in the present study to evaluate the human inter-ethnic and inter-individual variation in lasiocarpine induced bioactivation that may result from differences in the kinetics of lasiocarpine metabolism in the liver. In our previous study (Ning et al. 2019), the fraction of blood flow to the small intestine for the Caucasian population was $18.1 \%$, obtained from Brown et al. (1997) which is the flow of the portal vein including the sum of the blood flow to stomach, esophagus, small intestine, large intestine, spleen, and pancreas. In the present study, the value of the fraction of blood flow to the small intestine for the Caucasian population was further refined to $10 \%$ as reported from ICRP (2003). It is important to note that this refinement will only have a minor influence on the predicted $C_{\max }$ of lasiocarpine in liver blood. In the present study, we further included the kinetic parameters of 7-GS-DHP formation in the PBK model code to determine the relative inter-ethnic and inter-individual differences in bioactivation of lasiocarpine. As an example the equation for the liver is described as follows:

Amount of lasiocarpine in liver tissue $=\mathrm{AMLB}_{(\mathrm{in})}+\mathrm{AMLSi}_{(\mathrm{in})}$ $-\mathrm{AMLB}_{\text {(out) }}-\left(\mathrm{AMLM1}^{\prime}-\mathrm{AMLM}^{\prime}\right)-\mathrm{AMLM2}$,

where $\mathrm{AMLB}_{(\mathrm{in})}$ is the total amount of lasiocarpine entering the liver from the blood (without amount via blood from the small intestine) $(\mu \mathrm{mol} / \mathrm{h})$, AMLSi $_{(\mathrm{in})}$ is the amount of lasiocarpine entering the liver via blood from the small intestine $(\mu \mathrm{mol} / \mathrm{h}), \mathrm{AMLB}_{(\mathrm{out})}$ reflects the amount of lasiocarpine leaving the liver to the systemic blood circulation $(\mu \mathrm{mol} / \mathrm{h})$, AMLM1' is the total amount of lasiocarpine metabolized in the liver $(\mu \mathrm{mol} / \mathrm{h})$, AMLM2' is the amount of 7-GS-DHP formed in the liver $(\mu \mathrm{mol} / \mathrm{h})$. The model code and physiological parameters can be found in the supplementary materials 3 . With this equation, the change of lasiocarpine in the liver tissue by metabolism is solely based on the lasiocarpine depletion as measured in vitro, while next to that, 7-GS-DHP formation in the liver is modelled as an additional parameter. It is important to note that the amount of 7-GS-DHP formation formally is already included in the AMLM1', which is the parameter describing clearance of lasiocarpine and this is why AMLM2', the formation of 7-GS-DHP is both added and subtracted. Adding it in this way allows quantification of the 7-GS-DHP formation without disturbing the mass balance.

\section{Sensitivity analysis}

A sensitivity analysis was performed to identify model parameters that influence the predicted 7-GS-DHP formation by the liver expressed in $\mu \mathrm{g} / \mathrm{kg}$ bw, $24 \mathrm{~h}$ after a single oral dose of $8 \mathrm{ng} / \mathrm{kg}$ bw/day and $3 \mathrm{mg} / \mathrm{kg}$ bw/day representing respectively an average daily intake of PAs via consumption of herbal tea (BfR 2013) and a dose level known to cause liver toxicity in humans (Culvenor 1983). Normalized sensitivity coefficients (SC) were calculated using the following equation:

$\mathrm{SC}=\left(C^{\prime}-C\right) /\left(P^{\prime}-P\right) \times(P / C)$,

where $C$ is the initial value of the model output (amount of 7-GS-DHP formation by the liver); $C^{\prime}$ is the modified model output resulting from a $5 \%$ increase of the parameter value; $P$ is the initial parameter value; $P^{\prime}$ is the modified parameter value (Evans and Andersen 2000).

\section{Individual PBK models and Monte Carlo simulation}

The kinetic constants shown to be of influence in the sensitivity analysis were determined from the incubation 
experiments with 25 individual Chinese male and 25 individual Caucasian male liver microsomes and were integrated into the PBK model resulting in 50 separate PBK models. Parameters that were not influential on the model outcome $(\mathrm{SC}<10.5 \mid)$ were kept as they were originally defined for the average Caucasian or average Chinese population. With the PBK models, the formation of 7-GS-DHP by the liver at an oral dose level of $8 \mathrm{ng} / \mathrm{kg}$ bw was predicted for each individual. Based on the incubations with 25 individual Chinese and 25 individual Caucasians, the mean and the $\mathrm{CV}$ of the $V_{\max }$ and $K_{\mathrm{m}}$ for lasiocarpine depletion and 7-GS-DHP formation were obtained.

Then, a Monte Carlo simulation was performed in connection with the PBK model to simulate the variation in the 7-GS-DHP formation by the liver that could occur in the Chinese, the Caucasian and the two populations combined at an oral dose of $8 \mathrm{ng} / \mathrm{kg}$ bw. For the Monte Carlo analysis, a total of 15000 simulations were performed, where in each simulation, the $V_{\max }$ and $K_{\mathrm{m}}$ of lasiocarpine depletion and of 7-GS-DHP formation were randomly taken from a log-normal distribution. The distributions were truncated at \pm 3 SD by excluding individuals with kinetic constants that were three times higher or lower than the geometric mean (GM) values from the Monte Carlo simulation (Punt et al. 2016; Strikwold et al. 2017). The kinetic constants for the lasiocarpine depletion and 7-GS-DHP formation in the liver were assumed to vary independently, but the maximum allowed ratio of the $V_{\max }$ of 7-GS-DHP formation and the $V_{\max }$ of lasiocarpine depletion was set to 1.0 to assure 7-GSDHP formation cannot exceed the total lasiocarpine depletion. The mean, $\mu_{\mathrm{w}}$ and standard deviation $\sigma_{\mathrm{w}}$ describing the log-normal distribution of $V_{\max }$ and $K_{\mathrm{m}}$ values were derived using the following equation (Zhang et al. 2007):

$\mu_{\mathrm{w}}=\ln \left(\mu_{x} / \sqrt{1+\mathrm{CV}_{x}^{2}}\right)$ and $\sigma_{\mathrm{w}}^{2}=\ln \left(1+\mathrm{CV}_{x}^{2}\right)$,

where $\mu_{x}$ is the mean of $V_{\max }$ or $K_{\mathrm{m}}$ and $\mathrm{CV}_{x}$ is the coefficient of variation for each of the values. The mean and CV of the $V_{\max }$ and $K_{\mathrm{m}}$ for lasiocarpine depletion and 7-GS-DHP formation were obtained in the present study based on the incubations with 25 individual Chinese male and 25 individual Caucasian male liver microsomes. The variation of 7-GSDHP formation obtained from the Monte Carlo simulations for the Chinese, the Caucasian and the two populations combined were compared to each other and to the predicted 7-GS-DHP formation from the individual PBK models for the 25 Chinese individuals, 25 Caucasian individuals and 50 individuals, respectively at the same oral dose level.

In the Monte Carlo simulations, the kinetic constants $V_{\max }$ and $K_{\mathrm{m}}$ of lasiocarpine depletion and of 7-GS-DHP formation were randomly and independently taken from their respective log-normal distributions. However, a relatively high and statistically significant correlation (Pearson $r$ of 0.83 ) between $V_{\max }$ of lasiocarpine depletion and of 7-GSDHP formation derived from the 50 human individuals was observed. There was also a strong correlation with Pearson $r$ of 0.88 between the catalytic efficiency of lasiocarpine depletion and of 7-GS-DHP formation (see supplementary materials 4). This correlation indicates that when clearance is effective, 7-GS-DHP formation is effective as well, indicating that treating them as independent parameters in the Monte Carlo simulation could possibly result in unrealistic estimates of the inter-individual differences. With Berkeley Madonna version (8.0.1), covariation between parameters cannot be analyzed. Instead, to include the effect of covariation in our model predictions with our Monte Carlo simulations using Berkeley Madonna, we allowed the ratio of the $V_{\max }$ of 7-GS-DHP formation and the $V_{\max }$ of lasiocarpine depletion in each simulation to only vary between the lowest and the highest observed values for these $V_{\max }$ ratios in the in vitro incubations from the 25 Chinese and 25 Caucasian individuals, which were 0.017 (derived from Chinese male individual number 20) and 0.96 (derived from Caucasian male individual 098H0150), respectively.

The model predictions with the Monte Carlo simulation were performed in the Berkeley Madonna software with version of 8.0.1 (Macey and Oster, UC Berkeley, CA, USA) applying Rosenbrock's algorithms for solving stiff systems. The output of the Monte Carlo simulations was statistically analyzed with GraphPad (GraphPad Prism software version 5.04, San Diego California USA) to obtain the different percentiles, i.e. GM, 90th and 99th percentile of the amount of 7-GS-DHP formed by the liver of the Chinese, the Caucasian and the two populations combined. In both the scenario of Monte Carlo simulation in which the $V_{\max }$ of 7-GS-DHP formation and the $V_{\max }$ of lasiocarpine depletion was set to 1.0, and the scenario to evaluate the effect of covariation, the CSAFs were obtained by dividing the 90th or 99th percentile of 7-GS-DHP formation by the GM (IPCS 2005).

\section{BMD analysis of predicted in vivo dose-response data obtained from the PBK-based reverse dosimetry approach}

In our previous study (Ning et al. 2019), the in vitro concentration-response curves for cytotoxicity obtained in pooled (5 donors) human hepatocytes were translated to in vivo dose-response curves for acute hepatotoxicity of lasiocarpine by PBK modelling-based reverse dosimetry. This quantitative in vitro to in vivo extrapolation (QIVIVE) was performed based on the $C_{\max }$ (the maximum concentration in liver blood). For the QIVIVE, each free concentration of lasiocarpine in the cell culture medium of the cytotoxicity experiments, was set equal to the free $C_{\max }$ of lasiocarpine 


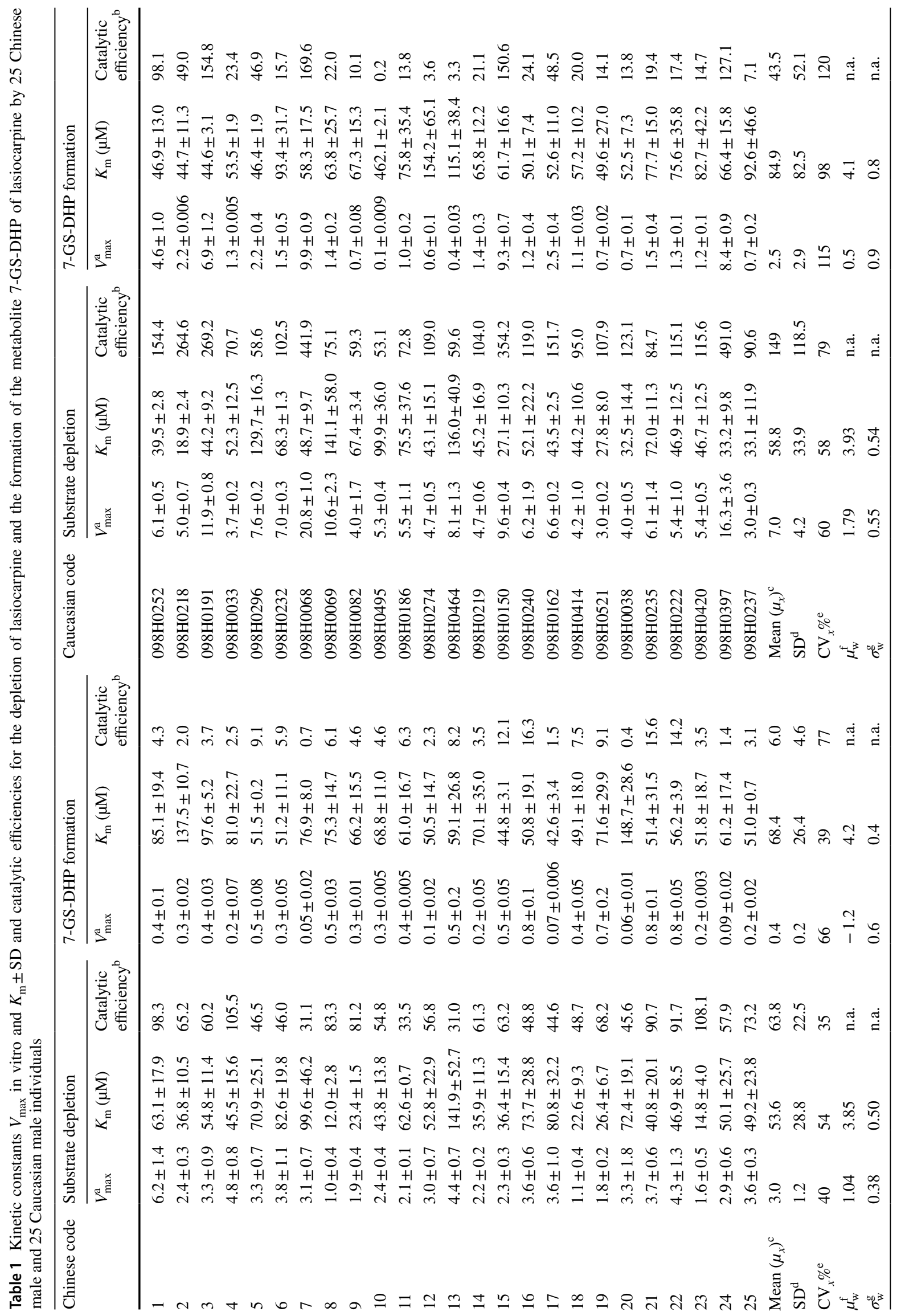


in the liver blood in the PBK model to calculate the oral dose that results in this concentration (Ning et al. 2019). In the present study, a similar approach was applied to define the dose-response curve for the average population, while the dose-response curves of the sensitive individuals (90th and 99th percentile) in the Chinese, the Caucasian and the two populations combined, were derived by applying the obtained CSAF to the dose-response curve of the average populations. The dose-response curve for the average population and the $\mathrm{BMDL}_{5}-\mathrm{BMDU}_{5}$ (the lower/upper confidence limit of the benchmark dose resulting in 5\% extra effect above background values) values for the acute liver toxicity of lasiocarpine for the sensitive individuals in the Chinese, Caucasian and the two populations combined were derived with the Hill model for continuous data by the PROAST software version 38.9 developed by RIVM (the Dutch National Institute for Public Health and the Environment) (Slob 2002).

\section{Results}

\section{Depletion of lasiocarpine by individual human liver microsomes}

Table 1 shows the kinetic constants $V_{\max }$ and $K_{\mathrm{m}}$, and the catalytic efficiency for depletion of lasiocarpine by 25 Chinese male and 25 Caucasian male individuals, derived from experiments with liver microsomal incubations with the cofactor NADPH at increasing concentrations of lasiocarpine. For the 25 Chinese male individuals, the difference between the highest and the lowest catalytic efficiency amounted to 3.5 -fold with a CV of $35 \%$. For the 25 Caucasian male individuals, the difference between the highest and the lowest catalytic efficiency amounted to 9.2-fold with a CV of $79 \%$. This relatively high CV is mainly caused by Caucasian individuals 098H068 and 098H0397 which have relatively high $V_{\max }$ and low $K_{\mathrm{m}}$ values resulting in catalytic efficiencies that are 3.0- and 3.6-fold higher respectively than the average catalytic efficiency. The average catalytic efficiencies of lasiocarpine depletion of the Chinese population and the Caucasian population were 1.7-fold lower and 1.4-fold higher, respectively than the average catalytic efficiency obtained when the data for the two populations were combined (25 Chinese male +25 Caucasian male individuals) (Table 2). The CVs for the catalytic efficiency of the Chinese population and the Caucasian populations were 2.5and 1.1-fold lower, respectively than the catalytic efficiency of $89 \%$ for the combined population.

For the inter-ethnic comparison, it can be noted that the average catalytic efficiency for depletion of lasiocarpine was 2.3-fold lower in the Chinese population than in the Caucasian population, which is mainly due to the a 2.3 -fold lower 
Table 2 Descriptive statistics of the kinetic constants $V_{\max }$ in vitro and $K_{\mathrm{m}}$ and catalytic efficiencies for the depletion of lasiocarpine and the formation of the metabolite 7-GS-DHP from lasiocarpine by the two populations combined (50 human male individuals)

\begin{tabular}{|c|c|c|c|c|c|c|}
\hline & \multicolumn{3}{|c|}{ Lasiocarpine depletion } & \multicolumn{3}{|c|}{ 7-GS-DHP formation } \\
\hline & $V_{\max }^{\mathrm{a}}$ & $K_{\mathrm{m}}(\mu \mathrm{M})$ & $\begin{array}{l}\text { Catalytic } \\
\text { efficiency }\end{array}$ & $V_{\max }^{\mathrm{a}}$ & $K_{\mathrm{m}}(\mu \mathrm{M})$ & $\begin{array}{l}\text { Catalytic } \\
\text { efficiency }\end{array}$ \\
\hline Mean $\left(\mu_{x}\right)^{\mathrm{c}}$ & 5.0 & 56.2 & 106.5 & 1.4 & 76.7 & 24.7 \\
\hline $\mathrm{SD}^{\mathrm{d}}$ & 3.6 & 31.2 & 94.8 & 2.3 & 61.1 & 41.2 \\
\hline $\mathrm{CV}_{x} \%^{\mathrm{e}}$ & 73 & 56 & 89 & 161 & 80 & 167 \\
\hline$\mu_{\mathrm{w}}^{\mathrm{f}}$ & 1.4 & 3.9 & n.a. & -0.3 & 4.1 & n.a. \\
\hline$\sigma_{\mathrm{W}}^{\mathrm{g}}$ & 0.7 & 0.5 & n.a. & 1.1 & 0.7 & n.a. \\
\hline
\end{tabular}

Data for the Chinese and Caucasian population as such are presented in Table 1

n.a. not available

${ }^{\mathrm{a}} \mathrm{nmol} / \mathrm{min} / \mathrm{mg}$ liver microsomes for lasiocarpine depletion and for 7-GS-DHP formation

${ }^{\mathrm{b}} \mu \mathrm{l} / \mathrm{min} / \mathrm{mg}$ liver microsomes for lasiocarpine depletion and for 7-GS-DHP formation

${ }^{\mathrm{c}}$ Mean of kinetic constants $V_{\max }$ and $K_{\mathrm{m}}$, and catalytic efficiency for lasiocarpine depletion and 7-GS-DHP formation derived from 50 individuals ( 25 Chinese male individuals +25 Caucasian male individuals) as shown in Table 1

${ }^{\mathrm{d}}$ Standard deviation of kinetic constants $V_{\max }$ and $K_{\mathrm{m}}$, and catalytic efficiency for lasiocarpine depletion and 7-GS-DHP formation derived from 50 individuals ( 25 Chinese male individuals +25 Caucasian male individuals) as shown in Table 1

${ }^{\mathrm{e}}$ Coefficient variation $\%=\mathrm{SD} /$ mean $\left(\mu_{x}\right) \times 100 \%$

${ }^{\mathrm{f}}$ The mean for describing the $\log$-normal distribution $\mu_{\mathrm{w}}=\ln \left[\mu_{x} / \operatorname{sqrt}\left(1+\mathrm{CV}_{x}^{2}\right)\right]$

${ }^{\mathrm{g}}$ The standard deviation for describing the log-normal distribution $\sigma_{\mathrm{w}}=\operatorname{sqrt}\left[\ln \left(1+\mathrm{CV}_{x}^{2}\right)\right]$ average $V_{\max }$ value obtained for the Chinese population. The average $K_{\mathrm{m}}$ value in both populations is comparable. The CV of the catalytic efficiency for lasiocarpine depletion was 2.3fold lower for the Chinese population than for the Caucasian population, indicating that the inter-individual variation is higher within the 25 Caucasian male individuals than within the 25 Chinese male individuals.

\section{Bioactivation of lasiocarpine to 7-GS-DHP by individual human liver microsomes}

Table 1 shows the kinetic constants $V_{\max }$ and $K_{\mathrm{m}}$ and the catalytic efficiency for 7-GS-DHP formation obtained for the 25 Chinese male and 25 Caucasian male individuals. For the 25 Chinese male individuals, the difference between the highest and the lowest catalytic efficiency amounted to 43.5-fold with a CV of 77\%. This relatively high CV value is partly due to Chinese male individuals 7 and 20 which both show an about 6.5-fold lower $V_{\max }$ value, and a 1.1- and 2.2fold higher $K_{\mathrm{m}}$ value, respectively, than the average kinetic constants for the 25 individuals, resulting in about 12 -fold lower catalytic efficiency for these individuals compared to the average value. For 7-GS-DHP formation by the liver microsomes from the 25 Caucasian male individuals, the difference between the highest and the lowest catalytic efficiency amounted to 792 -fold with a CV of $120 \%$. This large difference in catalytic efficiency for 7-GS-DHP formation within these 25 Caucasian individuals is partly due to the relatively low activity by Caucasian individual $098 \mathrm{H} 0495$ with a 25.4 -fold lower $V_{\max }$ and a 5.5-fold higher $K_{\mathrm{m}}$ than the average kinetic constants of the 25 Caucasian individuals, resulting in a 203-fold lower catalytic efficiency than the average value. The average catalytic efficiencies of 7-GSDHP formation of the Chinese population and the Caucasian population were 4.2-fold lower and 1.8-fold higher, respectively than the average catalytic efficiency obtained for the two populations combined (25 Chinese male and 25 Caucasian male individuals) (Table 2). The CVs of the catalytic efficiency of the Chinese population and the Caucasian populations were 2.2- and 1.4-fold lower, respectively than the catalytic efficiency of $167 \%$ for the combined human population.

For the 7-GS-DHP formation, the average catalytic efficiency obtained with the Chinese population is 7.3-fold lower than the average catalytic efficiency obtained with the Caucasian population which is mainly due to a 7.1-fold lower average $V_{\max }$ value for 7-GS-DHP formation in the Chinese population compared to the average $V_{\max }$ for the Caucasian population. The average $K_{\mathrm{m}}$ value for 7-GS-DHP formation differed only 1.2 -fold between the two populations. The CV of $77 \%$ and $120 \%$ for the catalytic efficiencies for 7-GS-DHP formation in the Chinese and Caucasian population respectively showed that the inter-individual differences in kinetics for 7-GS-DHP formation are relatively high in both populations. 


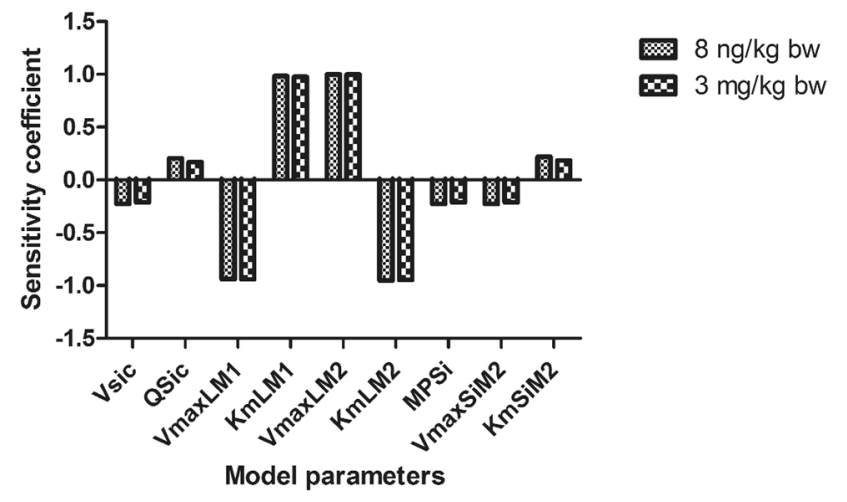

Fig. 2 Normalized sensitivity coefficients of PBK model parameters for the prediction of the formation of 7-GS-DHP by the liver expressed in $\mu \mathrm{g} / \mathrm{kg}$ bw, $24 \mathrm{~h}$ after oral administration of $8 \mathrm{ng} / \mathrm{kg}$ bw and $3 \mathrm{mg} / \mathrm{kg}$ bw of lasiocarpine. VSic fraction of small intestinal tissue, $Q S i c$ fraction of blood flow to small intestine, MPSi microsomal protein yield in small intestine. The $V_{\max } \mathrm{LM} 1$ and $V_{\max } \mathrm{SiM} 2$ represent the maximum rate of lasiocarpine depletion in the liver and small intestine, respectively. The $K_{\mathrm{m}} \mathrm{LM} 1$ and $K_{\mathrm{m}} \mathrm{SiM} 2$ are the MichaelisMenten constants for the lasiocarpine depletion in the liver and small intestine, respectively. $V_{\max } \mathrm{LM} 2$ and $K_{\mathrm{m}} \mathrm{LM} 2$ represent the maximum rate of formation and the Michaelis-Menten constant for the 7-GSDHP formation by the liver

\section{Sensitivity analysis}

The sensitivity analysis showed that the kinetic constants $V_{\max }$ and $K_{\mathrm{m}}$ for lasiocarpine depletion and 7-GSDHP formation by the liver of the Caucasian population influenced the predicted formation of 7-GS-DHP by the liver to a large extent (Fig. 2) both at a dose of $8 \mathrm{ng} / \mathrm{kg}$ bw and $3 \mathrm{mg} / \mathrm{kg}$ bw. The results of the sensitivity analysis performed in the Chinese population were similar to the results obtained in the Caucasian population (data not shown). Given that 7-GS-DHP formation reflects the potential bioactivation of lasiocarpine, the Monte Carlo simulation and CSAFs analysis were based on 7-GS-DHP formation by the liver.

\section{Individual PBK models and predicted 7-GS-DHP formation}

Figure 3 shows the PBK model based predictions for the time-dependent liver blood concentration of lasiocarpine at an oral dose of $8 \mathrm{ng} / \mathrm{kg}$ bw (Fig. 3a) and the dose-dependent formation of 7-GS-DHP (Fig. 3b) both for the average, and the most and least active of the 25 Chinese individuals, the 25 Caucasian individuals and the two populations combined ( 25 Chinese +25 Caucasians). The difference between the predicted $C_{\max }$ of lasiocarpine in liver blood for the individual with the highest and the lowest efficiency in lasiocarpine depletion is 2.5-fold for the Chinese population, 7.4fold for the Caucasian population and 12.7-fold for the two populations combined. Formation of 7-GS-DHP for each population was linear up to at least $80 \mathrm{mg} / \mathrm{kg}$ bw lasiocarpine. Figure 4 shows the box and whisker plots for the predicted formation of 7-GS-DHP by the liver at an oral dose a

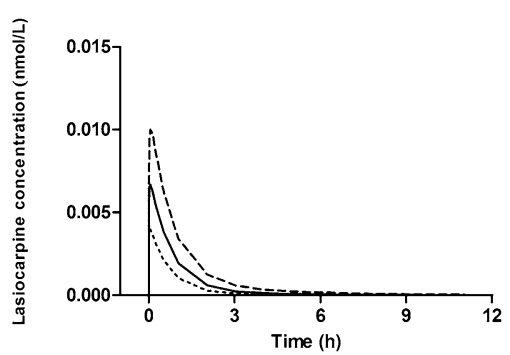

b

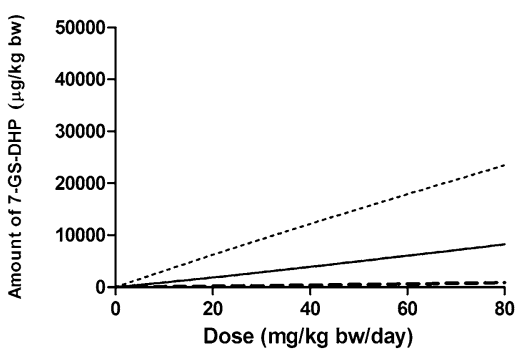

Caucasian population
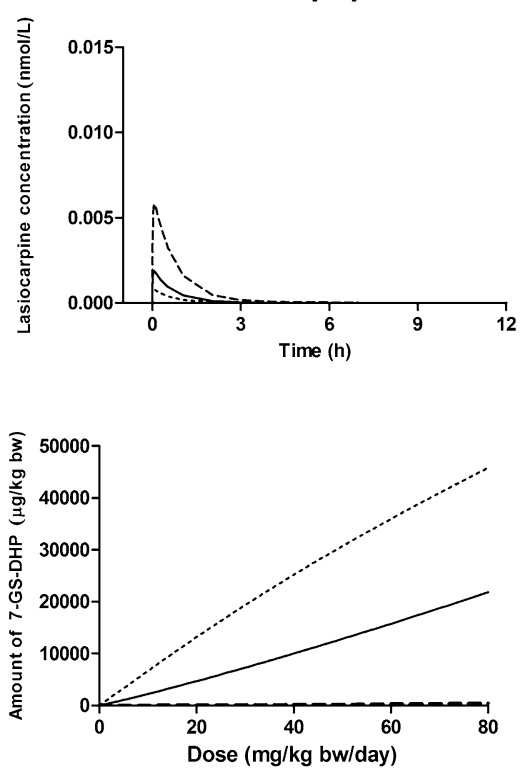

Two populations combined
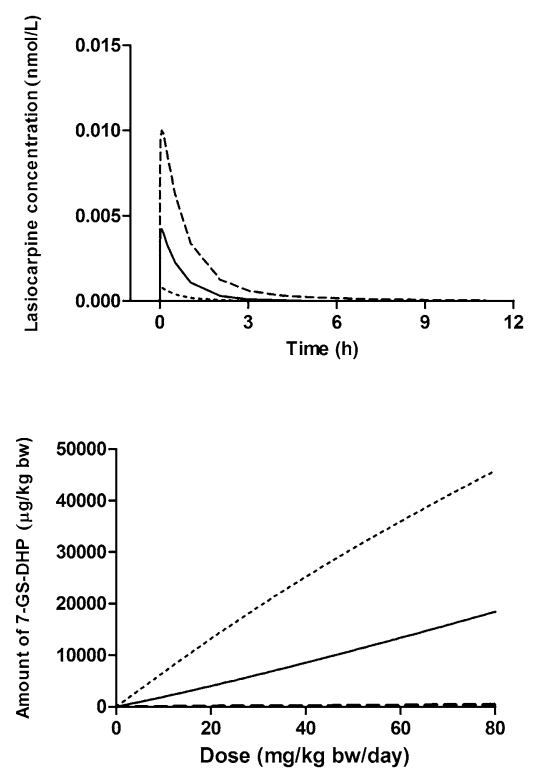

Fig. 3 PBK model predicted a time-dependent liver blood concentration of lasiocarpine at an oral lasiocarpine dose of $8 \mathrm{ng} / \mathrm{kg}$ bw and $\mathbf{b}$ dose-dependent formation of 7-GS-DHP by the liver, in the Chinese population, the Caucasian population and the two populations com- bined. The individuals with the highest (black dotted line), the lowest (black dashed line) and average (black solid line) efficiencies of lasiocarpine depletion and 7-GS-DHP formation are shown for each population 


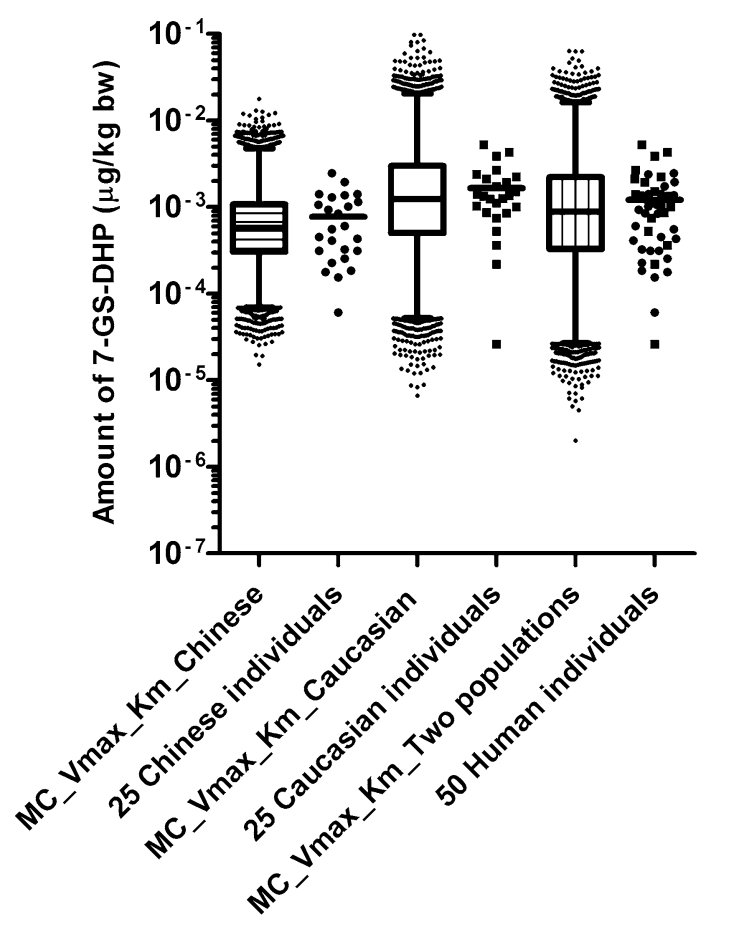

Fig. 4 Box and whisker plots representing the distribution of the predicted 7-GS-DHP formation by the liver, expressed in $\mu \mathrm{g} / \mathrm{kg}$ bw, $24 \mathrm{~h}$ after exposure to an oral lasiocarpine dose of $8 \mathrm{ng} / \mathrm{kg}$ bw that represents an average daily intake of PAs via consumption of herbal tea (BfR 2013) for 14,851 Chinese (box with horizontal lines), 13,119 Caucasians and 13,399 individuals from the two populations combined (box with vertical lines) obtained by Monte Carlo simulation including variation in $V_{\max }$ and $K_{\mathrm{m}}$ for lasiocarpine depletion and variation in $V_{\max }$ and $K_{\mathrm{m}}$ for 7-GS-DHP formation with the maximum allowed ratio of the $V_{\max }$ of 7-GS-DHP formation and the $V_{\max }$ of lasiocarpine depletion set as 1.0. The whiskers represent the $1 \mathrm{st}$ and 99th percentile of the populations and the small dots represent those values that were higher or lower than the 1st and 99th percentile of the studied populations consisting of less than about $2 \%$ of the 14,851 (Chinese), 13,119 (Caucasian) and 13,399 (two populations combined) Monte Carlo simulations. The figure also includes scatter plots representing the predicted amount of 7-GS-DHP formation by the liver $24 \mathrm{~h}$ after exposure to an oral lasiocarpine dose of $8 \mathrm{ng} / \mathrm{kg}$ bw for the 25 Chinese individuals (circles), the 25 Caucasian individuals (squares) and the 50 human individuals (circles + squares)

of $8 \mathrm{ng} / \mathrm{kg}$ bw for 25 Chinese individuals and 25 Caucasian individuals. Table 3 shows the GM and geometric CV of 7-GS-DHP formation predicted by individual PBK models in each population. For 7-GS-DHP formation, the difference between the highest and the lowest individual is 41 -fold for the Chinese population, 200-fold for the Caucasian population and 200-fold for the two populations combined. For the inter-ethnic comparison, the GM and geometric $\mathrm{CV}$ of the predicted 7-GS-DHP formation in 25 Chinese individuals is 2.2-fold lower and 1.1-fold higher, respectively than those values in 25 Caucasian individuals.

\section{Monte Carlo simulations and CSAF}

Table 3 shows the GM and geometric CV of 7-GS-DHP formation derived by Monte Carlo simulation in each population. For the Chinese population, the GM and geometric $\mathrm{CV}$ values obtained by the Monte Carlo simulation were comparable to the GM and geometric $\mathrm{CV}$ obtained with the 25 Chinese individuals. The Monte Carlo simulation results for both the Caucasian population and the two populations combined revealed that the GM values of 7-GS-DHP were comparable to the GM values obtained when analyzing the 25 Caucasian individuals and the 50 individuals, but that the geometric CV of the predicted 7-GS-DHP formation obtained from Monte Carlo simulation were about 3.0-fold higher than the values obtained from 25 Caucasian individuals and the 50 individuals. This indicates that the ethnic groups of 25 individuals each adequately represented the GM of 7-GS-DHP formation in a large population, but that the Monte Carlo simulation approach is more appropriate to identify sensitive individuals with respect to 7-GS-DHP formation within the population.

Figure 4 present the box and whisker plots for the predicted 7-GS-DHP formation by the liver obtained from the Monte Carlo simulations for the Chinese, the Caucasian and the two populations combined, applying a single oral dose of $8 \mathrm{ng} / \mathrm{kg}$ bw that represents an average daily intake of PAs via consumption of herbal tea (BfR 2013). Figure 5 presents the related frequency distribution for the predicted 7-GS-DHP formation by the liver for these modelled populations. The inter-ethnic comparison reveals that the GM of predicted 7-GS-DHP formation is 2.1-fold lower in the Chinese population than in the Caucasian population, while the differences in the predicted 7-GS-DHP formation between the populations amounted to 3.3- and 4.3-fold, respectively when the 90th and 99th percentile of populations were considered.

The CSAFs obtained by dividing the 90th or 99th percentile obtained with the maximum allowed ratio of the $V_{\max }$ of 7-GS-DHP formation and the $V_{\max }$ of lasiocarpine depletion set at 1.0, reflecting the worst-case scenario for the 7-GSDHP formation (IPCS 2005), are presented in Table 4. The CSAF values for the 99th percentile amounted to 8.3 for the Chinese population, 17.0 for the Caucasian population and 19.6 for the two populations combined, while these values were generally threefold lower in each population when the 90th percentile was used. Applying Monte Carlo simulation with the observed minimum and maximum acceptable boundary to evaluate the effect of modelling covariation on the predicted CSAF value, indicated that for each population the CSAF values were 1.1-fold (90th percentile and 99th percentile) lower than the CSAF values derived when using the maximum allowed ratio of 1.0 as the worst-case scenario (see Table 4). 
Table 3 Geometric mean, geometric mean CV\% and the fold difference between the minimum and maximum or the 1st and 99th percentile of the predicted formation of 7-GS-DHP by the liver $24 \mathrm{~h}$ after exposure to an oral lasiocarpine dose of $8 \mathrm{ng} / \mathrm{kg}$ bw by individual PBK models and in the population predicted by PBK model- ling linked with Monte Carlo simulation with the maximum allowed ratio of the $V_{\max }$ of 7-GS-DHP formation and the $V_{\max }$ of lasiocarpine depletion set at 1.0 in the Chinese population, the Caucasian population and two populations combined

\begin{tabular}{|c|c|c|c|c|c|c|}
\hline & \multicolumn{3}{|c|}{$\begin{array}{l}\text { 7-GS-DHP formation } \\
\text { Individual PBK models }\end{array}$} & \multicolumn{3}{|c|}{$\begin{array}{l}\text { 7-GS-DHP formation } \\
\text { Combination of PBK model with Monte Carlo simulation }\end{array}$} \\
\hline & $\begin{array}{l}\text { Chinese } \\
\text { population }\end{array}$ & $\begin{array}{l}\text { Caucasian } \\
\text { population }\end{array}$ & $\begin{array}{l}\text { Two populations } \\
\text { combined }\end{array}$ & $\begin{array}{l}\text { Chinese } \\
\text { population }\end{array}$ & $\begin{array}{l}\text { Caucasian } \\
\text { population }\end{array}$ & $\begin{array}{l}\text { Two populations } \\
\text { combined }\end{array}$ \\
\hline Geometric mean $(\mu \mathrm{g} / \mathrm{kg}$ bw) & 0.00054 & 0.0012 & 0.00079 & 0.00057 & 0.0012 & 0.00083 \\
\hline Geometric mean CV \% & 114 & 109 & 136 & 169 & 390 & 401 \\
\hline Fold difference & $41-$ fold $^{\mathrm{a}}$ & 200 -fold ${ }^{\mathrm{a}}$ & 200-fold ${ }^{\mathrm{a}}$ & 69-fold ${ }^{b}$ & 392-fold ${ }^{\mathrm{b}}$ & $607-$ fold $^{\mathrm{b}}$ \\
\hline
\end{tabular}

${ }^{a}$ Fold differences of the minimum and maximum of the predicted formation of 7-GS-DHP by individual PBK models

${ }^{b}$ Fold differences of the 1st and 99th percentile of the population for the formation of 7-GS-DHP obtained from the combination of PBK modelling with Monte Carlo simulations with the maximum allowed ratio of the $V_{\max }$ of 7-GS-DHP formation and the $V_{\max }$ of lasiocarpine depletion set at 1.0

\section{Predicted dose-response curve for the most sensitive population}

Figure 6 shows the predicted in vivo dose-response curves for the hepatotoxicity of lasiocarpine for the average and the most sensitive (90th and 99th percentile of the population) individuals of the Chinese, the Caucasian and the two populations combined. The in vivo dose-response curves for the most sensitive individuals were obtained by dividing the dose-response curves for the average population with the CSAF values for the 90th and 99th percentile of the population (using the CSAF values obtained with the maximum allowed ratio of the $V_{\max }$ of 7-GS-DHP formation and the $V_{\max }$ of lasiocarpine depletion set at 1.0). Table 5 shows the predicted $\mathrm{PoD}\left(\mathrm{BMDL}_{5}-\mathrm{BMDU}_{5}\right)$ values for the 90th percentile and 99th percentile of each population derived from these dose-response curves. The $\mathrm{BMDL}_{5}-\mathrm{BMDU}_{5}$ values for the 99th percentile of the Chinese, the Caucasian and the combined population were $1.8-5.0,0.4-1.4,0.8-1.9 \mathrm{mg} / \mathrm{kg}$ bw, while the $\mathrm{BMDL}_{5}-\mathrm{BMDU}_{5}$ values for the 90th percentile of each population were generally threefold higher in each population. Applying the CSAF values obtained with the observed minimum and maximum acceptable boundaries to evaluate the effect of modelling covariation on the dose-response curves for the most sensitive individuals in each population, the PoDs were up to 1.2-fold (90th and 99th) higher than the PoDs derived when using the maximum allowed ratio of 1.0.

For the ethnic comparison, the predicted PoD was 3- to 4-fold higher for the 90th and 99th percentile of Chinese the population than for the 90th and 99th percentile of the Caucasian population in both scenarios of the Monte Carlo simulations, indicating that Caucasians may be more sensitive to the liver toxicity of lasiocarpine than the Chinese population when taking the most sensitive individuals into account. For the most sensitive individuals within the two populations combined, the PoD values were up to twofold higher than the PoDs of the most sensitive individuals in the Caucasian population.

\section{Discussion}

The aim of the present study was to investigate the effect of the inter-individual and the inter-ethnic human kinetic variation in the Chinese and the Caucasian populations for lasiocarpine induced acute liver toxicity and bioactivation, by integrating PBK modelling with Monte Carlo simulations using human in vitro data, and to ultimately derive CSAFs. The obtained CSAFs, with two scenarios of Monte Carlo simulations, were further used to derive the concentration-response curves for the most sensitive individuals in each population, i.e. the 90th and 99th percentile of the population.

In the present study, the sensitivity analysis revealed that the 7-GS-DHP formation was highly affected by kinetic constants $V_{\max }$ and $K_{\mathrm{m}}$ of lasiocarpine depletion and the $V_{\max }$ and $K_{\mathrm{m}}$ of 7-GS-DHP formation and thus these parameters were included in the Monte Carlo simulation. Phase I metabolism of lasiocarpine, as shown in Fig. 1, is mediated by flavin-containing monooxygenases, carboxylesterases and cytochrome $\mathrm{P} 450$ enzymes. The P450 isoenzyme CYP $3 \mathrm{~A} 4$ is the primary metabolizing enzyme responsible for the metabolic activation of different types of PAs by human liver microsomes (Fashe et al. 2015; Fu et al. 2004; Li et al. 2011; Miranda et al. 1991). In the present study, the kinetic constants of depletion of lasiocarpine and 7-GS-DHP formation were derived from 25 male Chinese and 25 male Caucasian liver microsomes. It has been reported that male rodents are more sensitive to PA intoxication than female rodents. For 

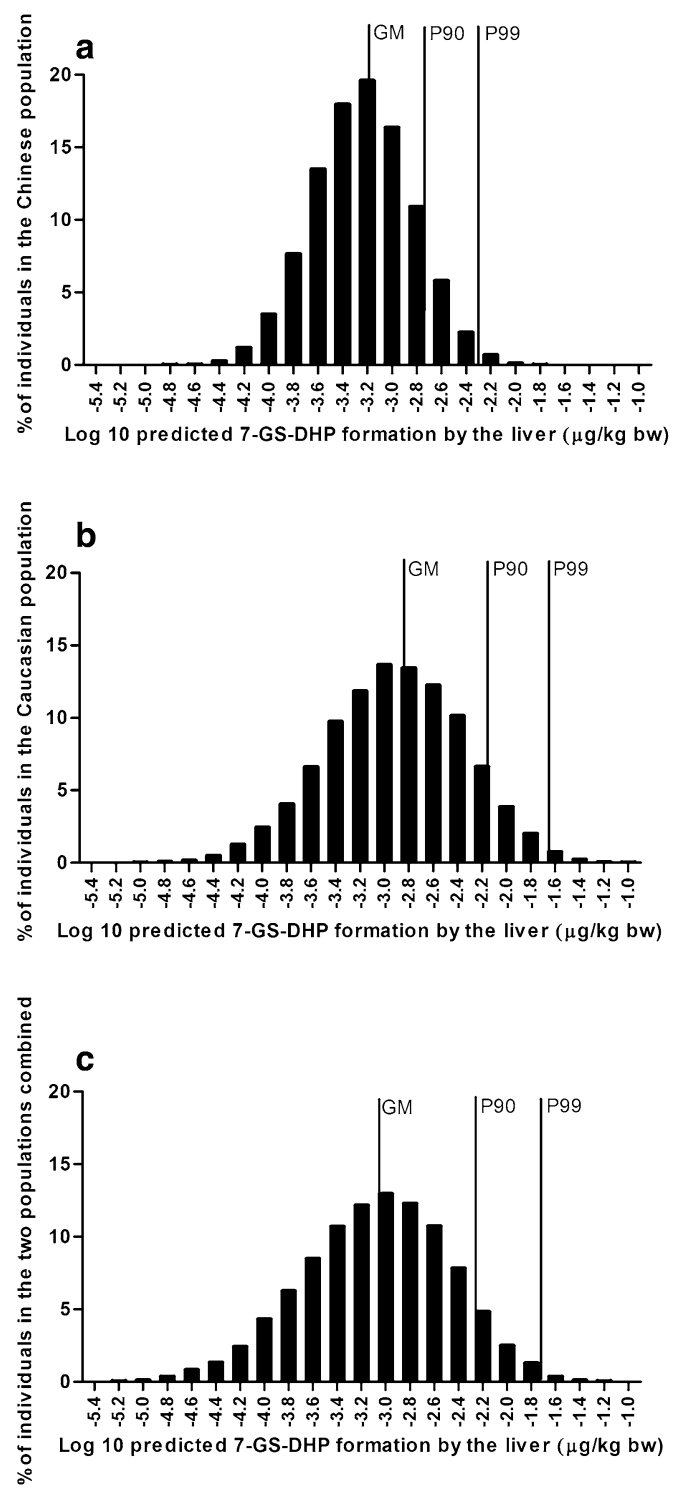

Fig. 5 Frequency distribution for the predicted total amount of 7-GSDHP formed by the liver $24 \mathrm{~h}$ after a single oral lasiocarpine dose of $8 \mathrm{ng} / \mathrm{kg}$ bw exposure, for 14,851 Chinese individuals (a), 13,119 Caucasian individuals (b) and 13,399 individuals in the two populations combined (c) obtained by Monte Carlo simulation including variation in the $V_{\max }$ and $K_{\mathrm{m}}$ for lasiocarpine depletion and for 7-GS-DHP formation with the maximum allowed ratio of the $V_{\max }$ of 7-GS-DHP formation and the $V_{\max }$ of lasiocarpine depletion set at 1.0. The GM, P90 and P99 represent the geometric mean, the 90th and 99th percentile of the distribution

humans, according to the review of Fu et al. (2004), there are no reports on a gender difference in the susceptibility of humans to pyrrolizidine alkaloid intoxication and recent reviews or studies regarding the gender difference in the sensitivity towards PAs are not available. However, many studies have shown that CYP 3A4 protein levels are about twofold higher in female livers than in male livers (Lamba et al. 2010; Nicolas et al. 2009; Schmidt et al. 2001; Wolbold et al. 2003; Yang et al. 2010; Zanger and Schwab 2013). In vivo, women had about $16 \%$ higher oral clearance and $20 \%$ higher systemic clearance rates than men for oral and intravenous administration of midazolam, a selective substrate of CYP 3A4 and CYP 3A5, but no significantly different area under the plasma concentration-time curve (AUC) of orally administered midazolam was observed between the sexes in a meta-analysis of ten studies on midazolam disposition including 409 healthy volunteers (Hu and Zhao 2010). However, several studies evaluating the gender difference in clearance of CYP 3A4 substrates have reported conflicting results (Cotreau et al. 2005; Nicolas et al. 2009; Schwartz 2003; Soldin and Mattison 2009), and thus so far no consensus has been reached regarding differences in the metabolism of CYP 3A4 substrates in man and women (Manoranjenni et al. 2012). Comparing the catalytic efficiency values of lasiocarpine depletion and 7-GS-DHP formation obtained using mixed gender Chinese liver microsomes (15 female +25 male) in our previous study (Ning et al. 2019), the average catalytic efficiency values for depletion of lasiocarpine and 7-GS-DHP formation derived from 25 Chinese male individuals is 1.4-fold lower and 1.5-fold higher than values from mixed gender Chinese liver microsomes, respectively. For Caucasians, the catalytic efficiency for lasiocarpine depletion and 7-GS-DHP formation obtained from 25 Caucasian male individuals is 1.3 -fold lower and 1.5-fold higher than those values derived from mixed gender Caucasian liver microsomes ( 16 female +17 male). Given that male individuals seem to be less efficient in lasiocarpine depletion but more active in bioactive metabolite formation, using the reported mean and CV of lasiocarpine depletion and 7-GS-DHP formation in the modelling approach applied in the present study will give insight in the most sensitive individuals for each population.

Besides the gender difference, other environmental factors such as age, smoking habits and alcohol consumption may affect CYP 3A4 activity. Rahmioglu et al. (2011) reported that about $20 \%$ of the inter-individual variations in induced CYP $3 \mathrm{~A} 4$ activity were associated with body mass index (BMI), alcohol use, and smoking habit, whereas age, diet and exercise levels were not associated with variations in CYP 3A4 activity. Hamilton et al. (2006) reported that in an analysis of thirty-two male Caucasians, $C_{\max }$ and AUC of erlotinib in plasma, metabolized in the human liver primarily by CYP $3 \mathrm{~A} 4$ but also by CYP $1 \mathrm{~A} 2$ in smokers, were about 1.5- and 2.8-fold respectively lower in non-smokers. Thus, it seems that smoking may enhance the CYP 3A4 activity. Table $\mathrm{S} 1$ shows the information on history of alcohol consumption and smoking habit which was only available for the 25 Caucasian individuals. Given the large interindividual variation in catalytic efficiency values of 7-GSDHP formation, and limited database regarding the history of alcohol consumption and smoking habit, conclusions on 
Table 4 The CSAFs obtained by 90th and 99th percentile for the Chinese population, the Caucasian population and the two populations combined in each scenario of Monte Carlo (MC) simulation

\begin{tabular}{|c|c|c|c|c|c|c|}
\hline \multirow[t]{2}{*}{ Monte Carlo scenario } & \multicolumn{3}{|c|}{ CSAFs at 90 th percentile } & \multicolumn{3}{|c|}{ CSAFs at 99th percentile } \\
\hline & $\begin{array}{l}\text { Chinese } \\
\text { population }\end{array}$ & $\begin{array}{l}\text { Caucasian } \\
\text { population }\end{array}$ & $\begin{array}{l}\text { Two popula- } \\
\text { tions combined }\end{array}$ & $\begin{array}{l}\text { Chinese } \\
\text { population }\end{array}$ & $\begin{array}{l}\text { Caucasian } \\
\text { population }\end{array}$ & $\begin{array}{l}\text { Two popula- } \\
\text { tions combined }\end{array}$ \\
\hline MC with the maximum allowed ratio of the $V_{\max }^{\mathrm{a}}$ & 3.3 & 5.2 & 5.7 & 8.3 & 17.0 & 19.5 \\
\hline $\begin{array}{l}\text { MC with observed minimum and maximum } \\
\text { acceptable boundaries }{ }^{\mathrm{b}}\end{array}$ & 3.3 & 5.2 & 5.3 & 8.3 & 16.8 & 17.6 \\
\hline
\end{tabular}

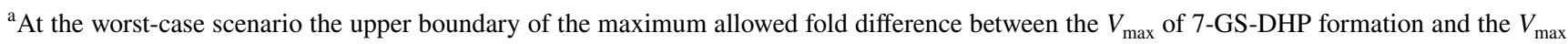
of lasiocarpine depletion was set at 1.0

${ }^{\mathrm{b}}$ The observed minimum and maximum acceptable boundaries were 0.017 and 0.96 calculated as the $V_{\max }$ of 7-GS-DHP formation divided by the $V_{\max }$ of lasiocarpine depletion derived from Chinese individual number 20 and Caucasian individual 098H0150, which showed the lowest and highest relative difference between the two $V_{\max }$ values, respectively

the association between PA metabolism and environmental factors have to await until further data become available in the future.

Previous studies have shown that CYP 3A4 plays an important role in PA metabolism, especially for the bioactivation pathway (Fashe et al. 2015; Li et al. 2011; Miranda et al. 1991). Inter-individual differences in CYP 3A4 abundance have been frequently reported. Rostami-Hodjegan and Tucker (2007) reported a CV of $119 \%$ of CYP 3A4 abundance in Caucasians by a meta-analysis and a CV of 87\% for CYP 3A4 abundance in Chinese (Inoue et al. 2006). Achour et al. (2014) reported that there were strong and significant correlations between the CYP 3A4 abundance in the liver and the CYP $3 \mathrm{~A}$ probe testosterone metabolism mediated by CYP 3A4 (spearman nonparametric correlation coefficient $r_{\mathrm{s}}=0.79, P<0.0001$ ) obtained by analysis of 23 individual liver microsomes from Caucasian subjects and one from an African American subject. Shu et al. (2001) also reported a good correlation (correlation coefficient of 0.79 ) between enzyme protein content and activity for CYP $3 \mathrm{~A} 4$ by analysis of 42 Chinese liver microsomes (26 Chinese male individuals +16 Chinese female individuals). The CYP 3A4 protein level was determined by Western blot analysis and the activity was measured by omeprazole sulfoxidation, mediated by CYP 3A4. In this study, the CV of CYP 3A4 activity in 42 Chinese individuals was reported to be $53.4 \%$ and gender difference in CYP 3A4 activity was not observed. Thus, for the liver, it can be speculated that the CV\% of CYP 3A4 activity in vitro is likely to have a comparable value to the CV\% value of CYP $3 \mathrm{~A} 4$ abundance in the liver. In the present study, the $\mathrm{CV}$ of catalytic efficiency of 7-GS-DHP formation in Chinese and Caucasian population were $77 \%$ and $120 \%$, respectively, which are comparable to the $\mathrm{CV}$ of $87 \%$ in Chinese and $119 \%$ in Caucasians of CYP 3A4 abundance reported in the literature (Inoue et al. 2006; Rostami-Hodjegan and Tucker 2007). The study of Rahmioglu et al. (2011) reported a high degree (66-88\%) of heritability in the CYP 3A4 inter-individual variation in twin studies as detected by repeated CYP 3A4-related drug administration, indicating that genetic polymorphism plays an important role in inter-individual variations in CYP 3A4 activity.

To date, 54 allelic variants of CYP 3A4 have been reported (https://www.pharmvar.org/), but only a few alleles are known to influence the CYP 3A4 expression or function. According to the literature (Wang et al. 2011), the CYP 3A4*22 mutation explains some of the genetic polymorphism showing a reduced activity in humans. For the inter-ethnic comparison, our previous study reported that the catalytic efficiencies of lasiocarpine depletion and of 7-GS-DHP formation in average pooled Chinese microsomes were 2.3- and 7.5-fold lower, respectively than those values obtained in average pooled Caucasian microsomes (Ning et al. 2019). The predicted $C_{\max }$ of lasiocarpine in the liver blood was twofold higher in the average Chinese than in the average Caucasians at similar dose levels. The present study showed similar results for the average catalytic efficiencies of lasiocarpine depletion and 7-GS-DHP formation in 25 Chinese individuals which were 2.3- and 7.3-fold lower, respectively compared to those values obtained in 25 Caucasian individuals. A review of McGraw and Waller (2012) reported that the CYP 3 A4*20 mutation has a relatively higher prevalence in the Chinese population $(22 \%)$ than in the Caucasian population (6\%). This allele has been shown to be devoid of any catalytic activity of CYP 3A4 in vitro (Westlind-Johnsson et al. 2006). The difference in CYP 3A4*20 prevalence between the two populations may explain the observed lower catalytic efficiency of lasiocarpine depletion and 7-GS-DHP formation in the Chinese population to some extent. However, CYP 3A4 *20 cannot fully explain the predicted phenotype difference between these two ethnic groups indicating that other SNPs and or factors may contribute as well. The genetic polymorphisms in CYP 3A4 appeared to be more prevalent in Caucasians than in Asian (Li and Bluth 2011). This seems to be in line with our results in the present paper that the coefficient of variation 

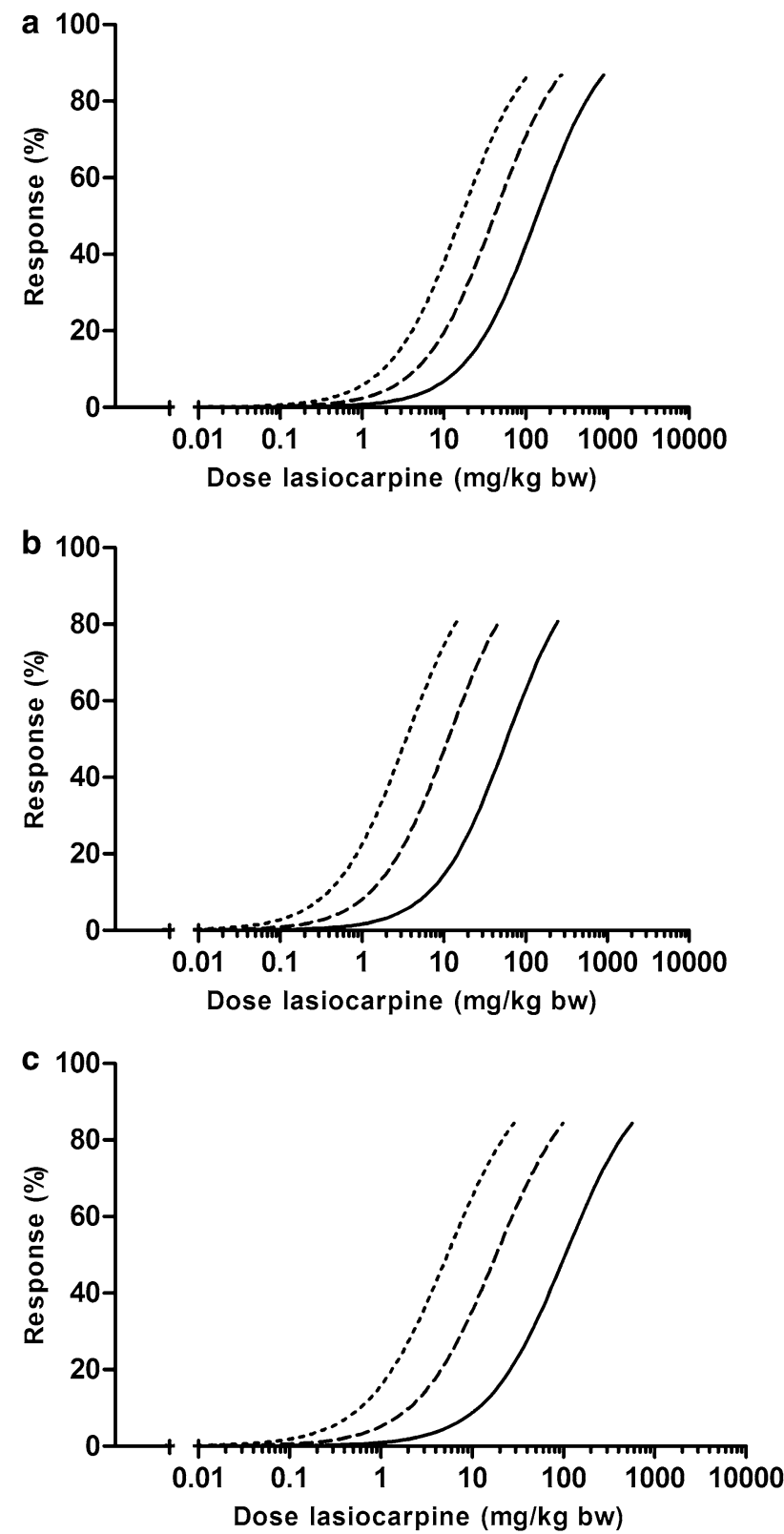

Fig. 6 Predicted dose-response curve for acute liver toxicity of lasiocarpine in the average (solid line), the 90th (dashed line) and 99th percentile (dotted line) of the Chinese (a), the Caucasian (b) and the two populations combined (c)

for 7-GS-DHP formation, was higher in the 25 Caucasian individuals than in the 25 Chinese individuals. A major bottleneck for characterizing CYP 3A activity is the lack of a specific probe to separate the CYP $3 \mathrm{~A} 4$ and CYP $3 \mathrm{~A} 5$ activity, hampering the identification of the association between genotype and phenotype (McGraw and Waller 2012). Thus, the present study showed that ethnic differences in CYP 3A4 activity exist, but identification of all the underlying factors that result in this CYP 3A4 related ethnic differences in the metabolic phenotype, remains a topic for future research.
Comparing CSAF values obtained using the 90th and the 99th percentile for the formation of 7-GS-DHP obtained from the Monte Carlo simulations, to the default uncertainty factor of 3.16 for inter-individual kinetic variation reveals that the default safety factor may not be adequately protective for lasiocarpine and/or PA toxicity in the studied populations. The IPCS document (IPCS 2005) stated that both inter-species and inter-individual variation in toxicokinetics would be ideally based on the free concentration of the reactive metabolite in the target tissue/organ. Based on this and the fact that the CSAFs derived from inter-individual variations for the formation of the metabolite 7-GS-DHP reflecting bioactivation are higher than the CSAF values generated from the variations in $C_{\max }$ of the parent compound in liver blood (shown in the supplementary materials 5), the use of the CSAF of 5.7 and 19.5 derived from the formation of 7-GS-DHP for the 90th percentile and 99th percentile of the two populations combined, respectively were considered as the worst-case scenario for risk assessment.

For compounds that are both genotoxic and carcinogenic but also have thresholded adverse endpoints like acute liver toxicity, such as lasiocarpine, a margin of exposure (MOE) approach can be used to perform a risk assessment for both the acute liver toxicity and the carcinogenicity. In the MOE approach, the PoD like a BMDL 10 (the lower confidence limit of the benchmark dose resulting in $10 \%$ extra effect above background values) is divided by the exposure level of the compound ( $\mathrm{mg} / \mathrm{kg}$ bw/day). In general, for a threshold endpoint such as acute liver toxicity, a MOE value of $>100$ is regarded to be of no or low concern for public health, while for evaluation of the carcinogenicity a MOE calculated using the $\mathrm{BMDL}_{10}$ for induction of liver tumors should be at least 10000 (EFSA 2015). Both values include (1) a factor 10 for inter-species differences including both kinetic (a factor of 4) and dynamic (a factor of 2.5) differences between experimental animals and humans and (2) a factor 10 for inter-individual differences in kinetics (a factor of 3.16) and dynamics (a factor of 3.16) within the human population (EFSA 2012, 2015). The factor of 10000 additionally incudes (3) a factor 10 for inter-individual differences in cell control and DNA repair; and (4) a factor 10 for the fact that the $\mathrm{BMDL}_{10}$ or the reference point is not identical to a no observed adverse effect level (NOAEL) (EFSA 2005). Our previous studies showed that the inter-species differences in acute liver toxicity of lasiocarpine amount to about twofold taking toxicokinetic and toxicodynamic differences into account (Chen et al. 2018; Ning et al. 2019). Based on the inter-species differences in toxicokinetics and toxicodynamics, together with a CSAF of 19.5 defined for the inter-individual kinetic differences for the two populations combined and the default factor of 3.16 for inter-individual dynamics differences, the compound specific MOE cut-off value for non-genotoxic effects would be $123(2 \times 3.16 \times 19.5)$, which 
Table 5 The predicted $\mathrm{BMDL}_{5}-\mathrm{BMDU}_{5}$ values for the acute liver toxicity of lasiocarpine for the 90th and 99th percentile of the Chinese, the Caucasian and the two populations combined obtained by CSAFs in each scenario of Monte Carlo simulation

\begin{tabular}{|c|c|c|c|c|c|c|}
\hline \multirow[t]{2}{*}{ Point of departures (mg/kg bw) } & \multicolumn{3}{|c|}{$\mathrm{BMDL}_{5}-\mathrm{BMDU}_{5}$ at 90 th percentile } & \multicolumn{3}{|c|}{$\mathrm{BMDL}_{5}-\mathrm{BMDU}_{5}$ at 99 th percentile } \\
\hline & $\begin{array}{l}\text { Chinese popu- } \\
\text { lation }\end{array}$ & $\begin{array}{l}\text { Caucasian } \\
\text { population }\end{array}$ & $\begin{array}{l}\text { Two populations } \\
\text { combined }\end{array}$ & $\begin{array}{l}\text { Chinese } \\
\text { population }\end{array}$ & $\begin{array}{l}\text { Caucasian } \\
\text { population }\end{array}$ & $\begin{array}{l}\text { Two popula- } \\
\text { tions combined }\end{array}$ \\
\hline $\begin{array}{l}\mathrm{BMDL}_{5}-\mathrm{BMDU}_{5} \text { values with the maximum } \\
\text { allowed ratio of the } V_{\max }^{\mathrm{a}}\end{array}$ & $4.4-12.5$ & $1.4-4.6$ & $2.7-6.7$ & $1.8-5.0$ & $0.4-1.4$ & $0.8-1.9$ \\
\hline $\begin{array}{l}\mathrm{BMDL}_{5}-\mathrm{BMDU}_{5} \text { values with observed mini- } \\
\text { mum and maximum acceptable boundaries }\end{array}$ & $4.4-12.5$ & $1.4-4.6$ & $2.7-7.0$ & $1.8-5.1$ & $0.4-1.4$ & $0.9-2.2$ \\
\hline
\end{tabular}

${ }^{\mathrm{a}} \mathrm{BMDL}_{5}-\mathrm{BMDU}_{5}$ values derived from dose-response curves obtained by CSAF with the worst-case scenario that the upper boundary was set to 1.0 as the maximum allowed fold difference between $V_{\max }$ of 7-GS-DHP formation and $V_{\max }$ of lasiocarpine depletion (Table 4)

${ }^{b} \mathrm{BMDL}_{5}-\mathrm{BMDU}_{5}$ values derived from dose-response curves obtained by CSAF with the observed minimum and maximum acceptable boundaries (Table 4)

is 1.2 -fold higher than the default threshold value of 100 used for inter- and intra-species differences. Evaluating the endpoint of carcinogenicity, the default value of 10000 for the MOE for genotoxic carcinogens could be increased to $12,324(19.5 \times 2 \times 3.16 \times 10 \times 10)$. When the CSAF obtained with the observed minimum and maximum acceptable boundaries, would be used, the compound specific MOE cutoff values for non-genotoxic effects and for genotoxic carcinogens would be 1.2-fold lower than these values derived when using the maximum allowed ratio of 1.0.

In conclusion, the present study shows that linking Monte Carlo simulations with PBK modelling can be used to predict the effect of inter-individual and inter-ethnic human kinetic variation in lasiocarpine induced acute liver toxicity, and that based on this CSAF values can be derived. The CSAFs obtained using the 90th and 99th percentile for inter-individual variation in the Chinese, the Caucasian and the two populations combined were higher than the default safety factor of 3.16 for inter-individual human differences in kinetics. The inter-ethnic human differences between the Chinese and the Caucasian population was predicted to be 2.1-, 3.3- and 4.3-fold when comparing the GM, the 90th percentile and the 99th percentile of the populations, respectively, showing that the Caucasian population is more sensitive than the Chinese population. Overall, the present study shows that integrating PBK modelling with Monte Carlo simulations is a powerful strategy to quantify interindividual variation in kinetics, which can be used to refine human risk assessment of PAs based on only human in vitro and in silico data.

Acknowledgements This work was funded by a grant from the China Scholarship Council (no. 201507720019 to NING JIA).

\section{Compliance with ethical standards}

Conflict of interest The authors declare that they have no conflict of interest.
Open Access This article is distributed under the terms of the Creative Commons Attribution 4.0 International License (http://creativeco mmons.org/licenses/by/4.0/), which permits unrestricted use, distribution, and reproduction in any medium, provided you give appropriate credit to the original author(s) and the source, provide a link to the Creative Commons license, and indicate if changes were made.

\section{References}

Achour B, Russell MR, Barber J, Rostami-Hodjegan A (2014) Simultaneous quantification of the abundance of several cytochrome P450 and uridine 5'-diphospho-glucuronosyltransferase enzymes in human liver microsomes using multiplexed targeted proteomics. Drug Metab Dispos 42:500-510

Bhat VS, Meek MEB, Valcke M, English C, Boobis A, Brown R (2017) Evolution of chemical-specific adjustment factors (CSAF) based on recent international experience; increasing utility and facilitating regulatory acceptance. Crit Rev Toxicol 47:733-753

Brown RP, Delp MD, Lindstedt SL, Rhomberg LR, Beliles RP (1997) Physiological parameter values for physiologically based pharmacokinetic models. Toxicol Ind Health 13:407-484

Bundesinstitut für Risikobewertung (BfR) (2013) Pyrrolizidine alkaloids in herbal teas and teas. BfR opinion

Chen L, Ning J, Louisse J, Wesseling S, Rietjens IMCM (2018) Use of physiologically based kinetic modelling-facilitated reverse dosimetry to convert in vitro cytotoxicity data to predicted in vivo liver toxicity of lasiocarpine and riddelliine in rat. Food Chem Toxicol 116:216-226

Cotreau MM, von Moltke LL, Greenblatt DJ (2005) The influence of age and sex on the clearance of cytochrome P450 3A substrates. Clin Pharmacokinet 44:33-60

Culvenor CC (1983) Estimated intakes of pyrrolizidine alkaloids by humans. A comparison with dose rates causing tumors in rats. J Toxicol Environ Health 11:625-635

European Food Safety Authority (EFSA) (2005) Opinion of the Scientific Committee on a request from EFSA related to a harmonised approach for risk assessment of substances which are both genotoxic and carcinogenic. EFSA J 3:282

European Food Safety Authority (EFSA) (2011) Scientific opinion on pyrrolizidine alkaloids in food and feed. EFSA J 9:2406

European Food Safety Authority (EFSA) (2012) Guidance on selected default values to be used by the EFSA Scientific Committee, Scientific Panels and Units in the absence of actual measured data. EFSA J 10:2579 
European Food Safety Authority (EFSA) (2015) Scientific opinion on acrylamide in food. EFSA J 13:4104

European Food Safety Authority (EFSA) (2017) Risks for human health related to the presence of pyrrolizidine alkaloids in honey, tea, herbal infusions and food supplements. EFSA J 15:4908

Evans MV, Andersen ME (2000) Sensitivity analysis of a physiological model for 2,3,7,8-tetrachlorodibenzo- $p$-dioxin (TCDD): assessing the impact of specific model parameters on sequestration in liver and fat in the rat. Toxicol Sci 54:71-80

Fashe MM, Juvonen RO, Petsalo A, Rasanen J, Pasanen M (2015) Species-specific differences in the in vitro metabolism of lasiocarpine. Chem Res Toxicol 28:2034-2044

Fu PP, Xia Q, Lin G, Chou MW (2004) Pyrrolizidine alkaloids-genotoxicity, metabolism enzymes, metabolic activation, and mechanisms. Drug Metab Rev 36:1-55

Hamilton M, Wolf JL, Rusk J, Beard SE, Clark GM, Witt K, Cagnoni PJ (2006) Effects of smoking on the pharmacokinetics of erlotinib. Clin Cancer Res 12:2166-2171

Hu ZY, Zhao YS (2010) Sex-dependent differences in cytochrome P450 $3 \mathrm{~A}$ activity as assessed by midazolam disposition in humans: a meta-analysis. Drug Metab Dispos 38:817-823

Inoue S, Howgate EM, Rowland-Yeo K, Shimada T, Yamazaki H, Tucker GT, Rostami-Hodjegan A (2006) Prediction of in vivo drug clearance from in vitro data. II: Potential inter-ethnic differences. Xenobiotica 36:499-513

International Agency for Research on Cancer (IARC) (2017) Agents classified by the IARC monographs, vols 1-118. International Agency for Research on Cancer, Lyon

International Commission on Radiological Protection (ICRP) (2003) Basic anatomical and physiological data for use in radiological protection reference values. ICRP publication 89. Ann ICRP $32: 3-4$

International Programme on Chemical Safety (IPCS) (1989) International programme on chemical safety, health and safety guide no 26: pyrrolizidine alkaloids health and safety guide. World Health Organization, Geneva

International Programme on Chemical Safety (IPCS) (2005) Chemical-specific adjustment factors for interspecies differences and human variability: guidance document for use of data in dose/ concentration-response assessment. WHO, Geneva

Kakar F, Akbarian Z, Leslie T, Mustafa ML, Watson J, van Egmond HP, Omar MF, Mofleh J (2010) An outbreak of hepatic venoocclusive disease in Western afghanistan associated with exposure to wheat flour contaminated with pyrrolizidine alkaloids. J Toxicol 2010:313280

Lamba V, Panetta JC, Strom S, Schuetz EG (2010) Genetic predictors of interindividual variability in hepatic CYP3A4 expression. J Pharmacol Exp Ther 332:1088-1099

Li J, Bluth MH (2011) Pharmacogenomics of drug metabolizing enzymes and transporters: implications for cancer therapy. Pharmgenom Pers Med 4:11-33

Li N, Xia QS, Ruan JQ, Fu PP, Lin G (2011) Hepatotoxicity and tumorigenicity induced by metabolic activation of pyrrolizidine alkaloids in herbs. Curr Drug Metab 12:823-834

Ma L, Zhao H, Xia Q, Cai L, Fu PP (2015) Synthesis and phototoxicity of isomeric 7,9-diglutathione pyrrole adducts: formation of reactive oxygen species and induction of lipid peroxidation. J Food Drug Anal 23:577-586

Manoranjenni C, Donald M, Amin R-H (2012) Sex differences in the clearance of CYP3A4 substrates: exploring possible reasons for the substrate dependency and lack of consensus. Curr Drug Metab 13:778-786

McGraw J, Waller D (2012) Cytochrome P450 variations in different ethnic populations. Expert Opin Drug Metab Toxicol 8:371-382

Medinsky MA, Leavens TL, Csanady GA, Gargas ML, Bond JA (1994) In vivo metabolism of butadiene by mice and rats: a comparison of physiological model predictions and experimental data. Carcinogenesis 15:1329-1340

Miranda CL, Reed RL, Guengerich FP, Buhler DR (1991) Role of cytochrome P450IIIA4 in the metabolism of the pyrrolizidine alkaloid senecionine in human liver. Carcinogenesis 12:515-519

National Toxicology Program (NTP) (1978) Bioassay of lasiocarpine for possible carcinogenicity. Natl Cancer Inst Carcinog Tech Rep Ser 39:1-66

Nicolas JM, Espie P, Molimard M (2009) Gender and interindividual variability in pharmacokinetics. Drug Metab Rev 41:408-421

Ning J, Chen L, Strikwold M, Louisse J, Wesseling S, Rietjens IMCM (2019) Use of an in vitro-in silico testing strategy to predict interspecies and inter-ethnic human differences in liver toxicity of the pyrrolizidine alkaloids lasiocarpine and riddelliine. Arch Toxicol 93:801-818

Prakash AS, Pereira TN, Reilly PEB, Seawright AA (1999) Pyrrolizidine alkaloids in human diet. Mutat Res Genet Toxicol Environ Mutagen 443:53-67

Punt A, Paini A, Spenkelink A, Scholz G, Schilter B, van Bladeren PJ, Rietjens IMCM (2016) Evaluation of interindividual human variation in bioactivation and DNA adduct formation of estragole in liver predicted by physiologically based kinetic/dynamic and monte carlo modeling. Chem Res Toxicol 29:659-668

Rahmioglu N, Heaton J, Clement G, Gill R, Surdulescu G, Zlobecka K, Hodgkiss D, Ma Y, Hider RC, Smith NW, Ahmadi KR (2011) Genetic epidemiology of induced CYP3A4 activity. Pharmacogenet Genom 21:642-651

Rostami-Hodjegan A, Tucker GT (2007) Simulation and prediction of in vivo drug metabolism in human populations from in vitro data. Nat Rev Drug Discov 6:140-148

Schmidt R, Baumann F, Hanschmann H, Geissler F, Preiss R (2001) Gender difference in ifosfamide metabolism by human liver microsomes. Eur J Drug Metab Pharmacokinet 26:193-200

Schwartz JB (2003) The influence of sex on pharmacokinetics. Clin Pharmacokinet 42:107-121

Shu Y, Cheng ZN, Liu ZQ, Wang LS, Zhu B, Huang SL, Ou-Yang DS, Zhou HH (2001) Interindividual variations in levels and activities of cytochrome P-450 in liver microsomes of Chinese subjects. Acta Pharmacol Sin 22:283-288

Slob W (2002) Dose-response modelling of continuous endpoints. Tox Sci 66:298-312

Smith LW, Culvenor CC (1981) Plant sources of hepatotoxic pyrrolizidine alkaloids. J Nat Prod 44:129-152

Soldin OP, Mattison DR (2009) Sex differences in pharmacokinetics and pharmacodynamics. Clin Pharmacokinet 48:143-157

Stegelmeier BL, Edgar JA, Colegate SM, Gardner DR, Schoch TK, Coulombe RA, Molyneux RJ (1999) Pyrrolizidine alkaloid plants, metabolism and toxicity. J Nat Toxins 8:95-116

Strikwold M, Spenkelink B, Woutersen RA, Rietjens IMCM, Punt A (2017) Development of a combined in vitro physiologically based kinetic (PBK) and Monte Carlo modelling approach to predict interindividual human variation in phenol-induced developmental toxicity. Toxicol Sci 157:365-376

Wang D, Guo Y, Wrighton SA, Cooke GE, Sadee W (2011) Intronic polymorphism in CYP3A4 affects hepatic expression and response to statin drugs. Pharmacogenom J 11:274-286

Westlind-Johnsson A, Malmebo S, Johansson A, Otter C, Andersson $\mathrm{TB}$, Johansson I, Edwards RJ, Boobis AR, Ingelman-Sundberg M (2003) Comparative analysis of CYP3A expression in human liver suggests only a minor role for CYP3A5 in drug metabolism. Drug Metab Dispos 31:755-761

Westlind-Johnsson A, Hermann R, Huennemeyer A, Hauns B, Lahu G, Nassr N, Zech K, Ingelman-Sundberg M, von Richter O (2006) Identification and characterization of CYP3A4*20, a novel rare CYP3A4 allele without functional activity. Clin Pharmacol Ther 79:339-349 
Wilkinson GR (2005) Drug metabolism and variability among patients in drug response. N Engl J Med 352:2211-2221

Wolbold R, Klein K, Burk O, Nussler AK, Neuhaus P, Eichelbaum M, Schwab M, Zanger UM (2003) Sex is a major determinant of CYP3A4 expression in human liver. Hepatology 38:978-988

Yang X, Zhang B, Molony C, Chudin E, Hao K, Zhu J, Gaedigk A, Suver C, Zhong H, Leeder JS, Guengerich FP, Strom SC, Schuetz E, Rushmore TH, Ulrich RG, Slatter JG, Schadt EE, Kasarskis A, Lum PY (2010) Systematic genetic and genomic analysis of cytochrome $\mathrm{P} 450$ enzyme activities in human liver. Genome Res 20:1020-1036
Zanger UM, Schwab M (2013) Cytochrome P450 enzymes in drug metabolism: regulation of gene expression, enzyme activities, and impact of genetic variation. Pharmacol Ther 138:103-141

Zhang X, Tsang AM, Okino MS, Power FW, Knaak JB, Harrison LS, Dary CC (2007) A physiologically based pharmacokinetic/ pharmacodynamic model for carbofuran in Sprague-Dawley rats using the exposure-related dose estimating model. Toxicol Sci 100:345-359

Publisher's Note Springer Nature remains neutral with regard to jurisdictional claims in published maps and institutional affiliations. 